\begin{tabular}{|c|l|}
\hline Title & Theoretical study on the translation and rotation of an elliptic camphor particle \\
\hline Author(s) & Iida, Keita; Kitahata, Hiroyuki; Nagay ama, Masaharu \\
\hline Citation & $\begin{array}{l}\text { Physica. D, Nonlinear phenomena, 272, 39-50 } \\
\text { https://doi.org/40.1016j.physd.2014.01.005 }\end{array}$ \\
\hline Issue Date & 201404_01 \\
\hline Doc URL & http://hdl.handle.net/2115/56608 \\
\hline Type & article (author version) \\
\hline File Information & PhysicaD_272_39.pdf \\
\hline
\end{tabular}

Instructions for use 


\title{
Theoretical Study on the Translation and Rotation of an Elliptic Camphor Particle
}

\author{
Keita Iida $^{\mathrm{a}}$, Hiroyuki Kitahata ${ }^{\mathrm{b}, \mathrm{c}}$, Masaharu Nagayama ${ }^{\mathrm{d}, \mathrm{e}, *}$ \\ ${ }^{a}$ Graduate School of Medicine, Tohoku University, 2-1, Seiryo-machi, Aoba-ku, Sendai, 980-8575, Japan \\ ${ }^{b}$ Graduate School of Science, Chiba University, 1-33, Yayoi-cho, Inage-ku, Chiba, 263-8522, Japan \\ ${ }^{c}$ PRESTO, Japan Science and Technology Agency, 4-1-8, Honcho Kawaguchi, Saitama, 332-0012, Japan \\ ${ }^{d}$ Research Institute for Electronic Science, Hokkaido University, N20W10, Kita-Ward, Sapporo, 001-0020, Japan \\ ${ }^{e}$ CREST, Japan Science and Technology Agency, 4-1-8, Honcho Kawaguchi, Saitama, 332-0012, Japan
}

\begin{abstract}
The spontaneous motion of an elliptic camphor particle floating on water is studied theoretically and experimentally. Considering a mathematical model for the motion of an elliptic camphor particle in a two-dimensional space, we first investigate the asymptotic solutions with numerical computation. We then introduce a small parameter $\varepsilon$ into the definition of the particle shape, which represents an elliptic deformation from a circular shape and, by means of perturbation theory, we analytically calculate the travelling solution to within $O(\varepsilon)$. The results show that short-axis-directed travelling solutions primarily bifurcate from stationary solutions and that longaxis-directed ones are secondary which means that elliptic camphor particles are easier to move in the short-axis direction. Furthermore, we show that rotating solutions bifurcate from stationary solutions and that the bifurcation point changes with $O\left(\varepsilon^{2}\right)$, which suggests that elliptic camphor disks are easier to exhibit translational motion, rather than rotational, within the small deformation. Finally, our theoretical suggestions are confirmed by an experiment.
\end{abstract}

Keywords: Surfactant-water system, Reaction-diffusion system, Bifurcation theory, Perturbation theory

MSC: 00A71; 00A79; 35K57; 70E20

${ }^{*}$ Corresponding author. Tel.:+81-11-706-3357; fax:+81-11-706-2891

Email address: nagayama@es.hokudai.ac.jp (Masaharu Nagayama) 


\section{Introduction}

Spontaneous motion seen in the natural world has been an origin of fascination for many researchers. What attracts us are the various manners of these motions and relevant phenomena, such as spatiotemporal patterning. Examples include locomotion of motor proteins in living organisms [1], swimming motions of motile bacteria [2], the chemical locomotion of metallic nanorods in aqueous solutions [3], the spontaneous motion of surfactant particles atop liquid surfaces [4$6]$, spontaneous droplet motions [7-10] and propagating solitary waves in a vertically vibrating suspension [11].

To create a novel chemical motor that works efficiently under isothermal conditions, scientists have been taking a cue from biological motors which convert chemical energy into mechanical energy under almost isothermal and non-equilibrium conditions [12]. A so-called surfactant-water system has been introduced as a prototype of such a chemo-mechanical energy transducer. For example, an oil-water system in which an oil droplet exhibits vectorial motion in an aqueous solution was investigated in 1993 and 1996 [7, 12]. In this system, the interaction of the surfactant molecules in the aqueous solution and the iodide ions in the oil droplet imparts a surface instability on the droplet, by means of a surfactant molecular layer which changes the surface pressure. As a result, spontaneous motion and deformations are observed.

On the other hand, a camphor-water system in which a camphor particle exhibits unidirectional motions, such as rotation and translation, atop water was introduced [4]. Regarding this system, both experimental and theoretical studies showed that the spontaneous motion of surfactant particles is attributable to nonuniformity of surface tension around the particle [13, 14]. They also provided an important idea for realizing such nonuniformity; that is, the asymmetric design of the system, e.g., shape of surfactant particles, shape of the chamber, chemical concentration field, etc. Lately, a number of studies have given a variety of examples, along with an improved understanding of spontaneous motions $[8,9,15-18]$.

Considering the simple case in which a surfactant particle or droplet moves over an aqueous surface, one can see that an asymmetric surface tension profile is necessary for inducing motion. In fact, theoretical studies of a camphor-water system have shown that the symmetric profile of surface tension for a standing camphor disk can be unstable with respect to an infinitesimal perturbation, and then a motion in a certain direction is stabilized [14, 19]. Consequently, the surface concentration of camphor molecules becomes asymmetric with respect to the center of the camphor disk.

In the last decade, motions of surfactant particles, almost undeformable droplets, and that of "camphor boats" as well, have been investigated by means of mathematical modelling under certain conditions $[14,20,21]$. As mentioned above, they can explain the spontaneous symmetry breaking and many other phenomena, such as oscillatory motions in a linear chamber [13] and jamming in an annular channel [22-24]. Nevertheless, there are still many problems which are difficult to explain using their spatial one-dimensional model. One example is found in an alcoholwater system, in which an alcohol droplet with an appropriate volume deforms spontaneously due to the Marangoni effect and then exhibits vectorial motion over an aqueous surface [9]. The deformation of the droplet seems to drive the motion and vice versa, but the relationship between motion and deformation has not yet been fully understood. Another example is a translational 
motion coupled with rotation in a camphor-water system whose spatial two-dimensional model was introduced in 2005 [25]. However, so far, this model has been analyzed only for a circular disk, which cannot exhibit rotational motion [19].

Among these problems, we are interested in the relationship between the shape (or deformation) of surfactant particles (or droplets) and the spontaneous motion. One possible approach is to investigate the shape-dependent motion neglecting the dynamics of deformation. For this purpose, we consider the camphor-water system. The definition of the particle shape is based on recent research on the self-propelled motion of deformable domains. As in [26, 27], small deformation of a circular shape with a radius $r_{0}$ can be written as

$$
r(\theta)=r_{0}\left[1+\sum_{k=2}^{\infty}\left(a_{k} \cos k \theta+b_{k} \sin k \theta\right)\right],
$$

where $(r, \theta)$ are two-dimensional polar coordinates, and $a_{k}$ and $b_{k}$ are infinitesimally small parameters. Here, we choose the 2-mode deformation by dropping all $k$-mode terms except for $k=2$. This is the most fundamental, but nontrivial, deformation that is an elliptic deformation.

When considering the elliptic shape of a camphor particle, we face a simple question: in which direction does the elliptic camphor particle move? The present study provides us with an answer to this question by means of numerical and analytical investigations based on mathematical modelling, as well as on experimental observations.

\section{Mathematical model}

Based on previous papers $[14,25,28]$, we introduce a mathematical model for the spontaneous motion of a camphor particle atop a water surface. Let us consider a two-dimensional space $\Omega$ $\left(=\mathbb{R}^{2}\right)$ as the water surface and its subset $\Omega_{\mathrm{c}}$ as the interior of the camphor particle whose boundary is defined as follows:

$$
\partial \Omega_{\mathrm{c}}\left(\boldsymbol{x}_{\mathrm{c}}, \theta_{\mathrm{c}}\right)=\left\{\boldsymbol{x} \in \Omega \mid \boldsymbol{x}=\boldsymbol{x}_{\mathrm{c}}+\boldsymbol{p}\right\},
$$

where $\boldsymbol{x}_{\mathrm{c}}(t)\left(=\left(x_{\mathrm{c}}(t), y_{\mathrm{c}}(t)\right)\right)$ and $\theta_{\mathrm{c}}(t)$ are the center of mass and characteristic angle of the camphor particle, respectively, and $\boldsymbol{p}$ is the parametric representation for $\partial \Omega_{\mathrm{c}}$ (see Fig. 1). Let $u(t, \boldsymbol{x})$ be the surface concentration of the camphor molecular layer.

The model equations are as follows:

$$
\begin{gathered}
m \frac{\mathrm{d}^{2} \boldsymbol{x}_{\mathrm{c}}}{\mathrm{d} t^{2}}=-\eta_{\mathrm{t}} \frac{\mathrm{d} \boldsymbol{x}_{\mathrm{c}}}{\mathrm{d} t}+\boldsymbol{F}, \\
I \frac{\mathrm{d}^{2} \theta_{\mathrm{c}}}{\mathrm{d} t^{2}}=-\eta_{\mathrm{r}} \frac{\mathrm{d} \theta_{\mathrm{c}}}{\mathrm{d} t}+T, \\
\frac{\partial u}{\partial t}=D \Delta u-\alpha u+f\left(\boldsymbol{x}, \boldsymbol{x}_{\mathrm{c}}, \theta_{\mathrm{c}}\right),
\end{gathered}
$$




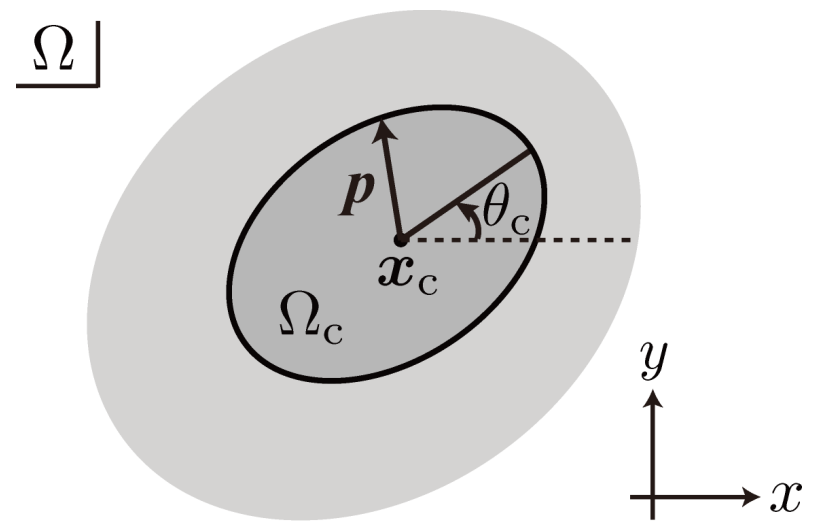

\section{: camphor molecular layer}

Fig. 1 Schematic representation of the camphor-water system in a two-dimensional space.

where $m$ and $I$ are the mass and moment of inertia of the camphor particle, $\eta_{\mathrm{t}}$ and $\eta_{\mathrm{r}}$ are the friction coefficients for translation and rotation, and $D$ and $\alpha$ are the diffusion coefficient and sublimation rate of the camphor molecular layer, respectively. In (3) and (4), $\boldsymbol{F}$ and $T$ are the driving force and torque exerted on the camphor particle due to the surface tension profile on $\partial \Omega_{\mathrm{c}}$, which are defined as follows:

$$
\begin{aligned}
& \boldsymbol{F}=\int_{\partial \Omega_{c}} \gamma(u) \boldsymbol{n} \mathrm{d} \ell, \\
& T=\int_{\partial \Omega_{c}} \gamma(u) \boldsymbol{p} \times \boldsymbol{n} \mathrm{d} \ell,
\end{aligned}
$$

where $\boldsymbol{n}$ is the outer unit normal vector on $\partial \Omega_{\mathrm{c}}$. The notation " $\times$ " denotes the two-dimensional vector product. The surface tension $\gamma$ is assumed to be a decreasing function of $u$. Based on the experimental measurements [29], we apply the Hill-type function as follows:

$$
\gamma(u)=\frac{\beta^{n} \gamma_{0}}{\beta^{n}+u^{n}}+\gamma_{1},
$$

where $\beta, \gamma_{0}$ and $\gamma_{1}$ are positive constants and $n$ is a positive integer. In (5), $f$ is given by

$$
f\left(\boldsymbol{x}, \boldsymbol{x}_{\mathrm{c}}, \theta_{\mathrm{c}}\right)= \begin{cases}f_{0}, & \boldsymbol{x} \in \bar{\Omega}_{\mathrm{c}}, \\ 0, & \boldsymbol{x} \in \Omega \backslash \bar{\Omega}_{\mathrm{c}},\end{cases}
$$

where $f_{0}$ is the supply rate of the camphor molecular layer and $\bar{\Omega}_{\mathrm{c}}$ is the closure of $\Omega_{\mathrm{c}}$. The initial conditions are

$$
\begin{gathered}
\left(\boldsymbol{x}_{\mathrm{c}}(0), \frac{\mathrm{d} \boldsymbol{x}_{\mathrm{c}}}{\mathrm{d} t}(0)\right)=\left(\boldsymbol{x}_{0}, \boldsymbol{v}_{0}\right), \\
\left(\theta_{\mathrm{c}}(0), \frac{\mathrm{d} \theta_{\mathrm{c}}}{\mathrm{d} t}(0)\right)=\left(\theta_{0}, \omega_{0}\right), \\
u(0, \boldsymbol{x})=0, \quad \boldsymbol{x} \in \Omega,
\end{gathered}
$$


and the boundary condition is

$$
\lim _{|\boldsymbol{x}| \rightarrow \infty} u(t, \boldsymbol{x})=0 .
$$

Finally, to guarantee the existence and uniqueness of the solutions, we consider a regularity condition as follows:

$$
u(t, \cdot) \in C^{1}(\Omega) .
$$

To normalize the system (2)-(14), we introduce the following dimensionless variables:

$$
\tilde{t}=\alpha t, \quad \tilde{\boldsymbol{x}}=\sqrt{\frac{\alpha}{D}} \boldsymbol{x}, \quad \tilde{\boldsymbol{x}}_{\mathrm{c}}(\tilde{t})=\sqrt{\frac{\alpha}{D}} \boldsymbol{x}_{\mathrm{c}}(t), \quad \tilde{\theta}_{\mathrm{c}}(\tilde{t})=\theta_{\mathrm{c}}(t), \quad \tilde{u}(\tilde{t}, \tilde{\boldsymbol{x}})=\frac{\alpha}{f_{0}} u(t, \boldsymbol{x}),
$$

and the notations:

$$
\begin{aligned}
& \tilde{\Omega}=\left\{\sqrt{\frac{\alpha}{D}} \boldsymbol{x} \mid \boldsymbol{x} \in \Omega\right\}, \quad \tilde{\Omega}_{\mathrm{c}}=\left\{\sqrt{\frac{\alpha}{D}} \boldsymbol{x} \mid \boldsymbol{x} \in \Omega_{\mathrm{c}}\right\}, \quad \tilde{\boldsymbol{p}}=\sqrt{\frac{\alpha}{D}} \boldsymbol{p}, \quad \tilde{\boldsymbol{n}}=\sqrt{\frac{\alpha}{D}} \boldsymbol{n}, \\
& \mathrm{d} \tilde{\ell}=\sqrt{\frac{\alpha}{D}} \mathrm{~d} \ell, \quad \tilde{m}=\int_{\tilde{\Omega}_{\mathrm{c}}} \mathrm{d} \tilde{\boldsymbol{x}}, \quad \tilde{I}=\int_{\tilde{\Omega}_{\mathrm{c}}}\left|\tilde{\boldsymbol{x}}-\tilde{\boldsymbol{x}}_{\mathrm{c}}\right|^{2} \mathrm{~d} \tilde{\boldsymbol{x}}, \quad \tilde{\eta}_{\mathrm{t}}=\frac{1}{\rho D} \eta_{\mathrm{t}}, \quad \tilde{\eta}_{\mathrm{r}}=\frac{\alpha}{\rho D^{2}} \eta_{\mathrm{r}}, \\
& \tilde{\beta}=\frac{\alpha}{f_{0}} \beta, \quad \tilde{\gamma}_{0}=\frac{1}{\alpha \rho D} \gamma_{0}, \quad \tilde{\gamma}_{1}=\frac{1}{\alpha \rho D} \gamma_{1},
\end{aligned}
$$

where $\rho$ is a constant expressing the surface density of the camphor particle. Removing the tilde $(\sim)$ from each character, we derive the dimensionless system of (2)-(14) as follows:

$$
\begin{gathered}
\partial \Omega_{\mathrm{c}}\left(\boldsymbol{x}_{\mathrm{c}}, \theta_{\mathrm{c}}\right)=\left\{\boldsymbol{x} \in \Omega \mid \boldsymbol{x}=\boldsymbol{x}_{\mathrm{c}}+\boldsymbol{p}\right\}, \\
m \frac{\mathrm{d}^{2} \boldsymbol{x}_{\mathrm{c}}}{\mathrm{d} t^{2}}=-\eta_{\mathrm{t}} \frac{\mathrm{d} \boldsymbol{x}_{\mathrm{c}}}{\mathrm{d} t}+\int_{\partial \Omega_{\mathrm{c}}} \gamma(u) \boldsymbol{n} \mathrm{d} \ell, \\
I \frac{\mathrm{d}^{2} \theta_{\mathrm{c}}}{\mathrm{d} t^{2}}=-\eta_{\mathrm{r}} \frac{\mathrm{d} \theta_{\mathrm{c}}}{\mathrm{d} t}+\int_{\partial \Omega_{\mathrm{c}}} \gamma(u) \boldsymbol{p} \times \boldsymbol{n} \mathrm{d} \ell, \\
\frac{\partial u}{\partial t}=\Delta u-u+f\left(\boldsymbol{x}, \boldsymbol{x}_{\mathrm{c}}, \theta_{\mathrm{c}}\right), \\
\gamma(u)=\frac{\beta^{n} \gamma_{0}}{\beta^{n}+u^{n}}+\gamma_{1}, \\
f\left(\boldsymbol{x}, \boldsymbol{x}_{\mathrm{c}}, \theta_{\mathrm{c}}\right)= \begin{cases}1, & \boldsymbol{x} \in \bar{\Omega}_{\mathrm{c}}, \\
0, & \boldsymbol{x} \in \bar{\Omega}_{\mathrm{c}},\end{cases}
\end{gathered}
$$




$$
\begin{gathered}
\left(\boldsymbol{x}_{\mathrm{c}}(0), \frac{\mathrm{d} \boldsymbol{x}_{\mathrm{c}}}{\mathrm{d} t}(0)\right)=\left(\boldsymbol{x}_{0}, \boldsymbol{v}_{0}\right), \\
\left(\theta_{\mathrm{c}}(0), \frac{\mathrm{d} \theta_{\mathrm{c}}}{\mathrm{d} t}(0)\right)=\left(\theta_{0}, \omega_{0}\right), \\
u(0, \boldsymbol{x})=0, \quad \boldsymbol{x} \in \Omega, \\
\lim _{|\boldsymbol{x}| \rightarrow \infty} u(t, \boldsymbol{x})=0, \\
u(t, \cdot) \in C^{1}(\Omega) .
\end{gathered}
$$

\section{Numerical results}

In this section, we numerically solve (15)-(25) assuming that the camphor particle has an elliptical shape as follows:

$$
\partial \Omega_{\mathrm{c}}\left(\boldsymbol{x}_{\mathrm{c}}, \theta_{\mathrm{c}}\right):=\left\{(x, y) \in \Omega \mid h\left(\boldsymbol{x}, \boldsymbol{x}_{\mathrm{c}}, \theta_{\mathrm{c}}\right)=0\right\}
$$

Here, we put

$$
h\left(\boldsymbol{x}, \boldsymbol{x}_{\mathrm{c}}, \theta_{\mathrm{c}}\right)=\frac{\hat{x}^{2}}{a^{2}}+\frac{\hat{y}^{2}}{b^{2}}-1, \quad(\hat{x}, \hat{y})=\left(x-x_{\mathrm{c}}, y-y_{\mathrm{c}}\right) R\left(\theta_{\mathrm{c}}\right),
$$

where $R$ is the rotation matrix given by

$$
R(\theta)=\left(\begin{array}{cc}
\cos \theta & -\sin \theta \\
\sin \theta & \cos \theta
\end{array}\right)
$$

For the numerical computation, we replace (24) with the following periodic boundary condition by letting $\Omega=\left\{(x, y) \mid 0 \leq x<L_{x}, 0 \leq y<L_{y}\right\}$ :

$$
\left\{\begin{array}{lll}
u(t,(0, y))=u\left(t,\left(L_{x}, y\right)\right), & \frac{\partial u}{\partial x}(t,(0, y))=\frac{\partial u}{\partial x}\left(t,\left(L_{x}, y\right)\right), & 0 \leq y<L_{y}, \\
u(t,(x, 0))=u\left(t,\left(x, L_{y}\right)\right), & \frac{\partial u}{\partial y}(t,(x, 0))=\frac{\partial u}{\partial y}\left(t,\left(x, L_{y}\right)\right), & 0 \leq x<L_{x},
\end{array} \quad t \geq 0\right.
$$

where $L_{x}$ and $L_{y}$ are taken to be much greater than unity [30]. Also, instead of (20) and (25), we use the following $C^{1}$ function as an approximation of (20):

$$
f\left(\boldsymbol{x}, \boldsymbol{x}_{\mathrm{c}}, \theta_{\mathrm{c}}\right) \approx \frac{1}{2}\left(1-\tanh \frac{h\left(\boldsymbol{x}, \boldsymbol{x}_{\mathrm{c}}, \theta_{\mathrm{c}}\right)}{\lambda}\right),
$$

where $\lambda$ is a small parameter.

First, we investigate the asymptotic numerical solutions of (16)-(26) by computing (16)-(19), (21)-(23), (26)-(28). In (21) and (22), we assume the following initial slight perturbation to the translation and rotation:

$$
\boldsymbol{v}_{0}=\left(\delta_{1}, \delta_{1}\right), \quad \omega_{0}=\delta_{2}
$$


where $\delta_{1}$ and $\delta_{2}$ are small positive parameters, and $\theta_{0}$ in (22) is set to be 0. Fig. 2(a) shows the mode diagram of the asymptotic solutions, in which we take $\eta_{\mathrm{t}}$ and $\eta_{\mathrm{r}}$ as control parameters, and Fig. 2(b) shows the profiles of $u$ for the solutions in Fig. 2(a). In Fig. 2, there are three types of solutions; the stationary state (i.e., stationary solution symbolized by SS) is shown in region A, the solution corresponding to steady translation in the short-axis direction (i.e., short-axis-directed travelling solution STS) in region B, and the steady rotation solution (i.e., rotating solution RS) in region $\mathrm{C}$. Solutions denoted by the cross $(x)$ are ambiguous ones, whose velocity or angular velocity continued oscillating with small but non-negligible amplitude.

(a)

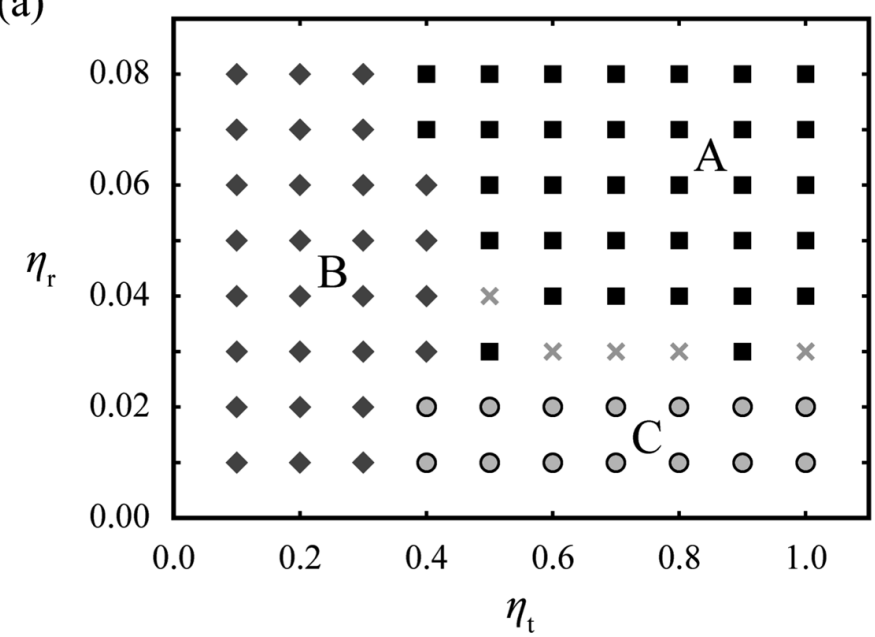

(b)

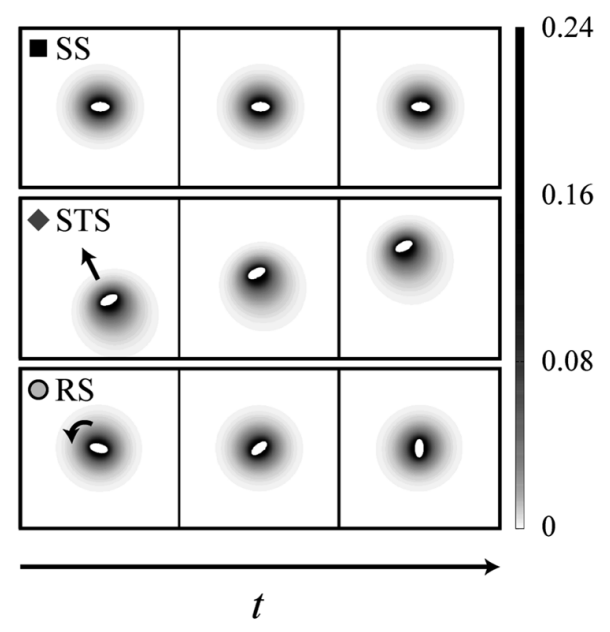

Fig. 2 Numerical results. (a) Mode diagram of the asymptotic solutions with respect to $\eta_{\mathrm{t}}$ and $\eta_{\mathrm{r}}$, in which the filled square denotes the stationary solution (SS), filled diamond the short-axisdirected travelling solution (STS), black-edged gray disk the rotating solution (RS) and cross the ambiguous solution. The letters A, B and C represent the parameter regions in which the solutions SS, STS and RS are observed, respectively. (b) Profiles of $u$ of the solutions SS, STS and RS. The gray level represents the magnitude of $u$ except for the interior of $\Omega_{\mathrm{c}}$, which is shown in the color bar on the right. The parameters are $\beta=0.4, \gamma_{0}=2.5, \lambda=0.01, a=1, b=0.5, \delta_{1}=0.1$ and $\delta_{2}=0.1$.

In Fig. 2(a), the appearance of $\mathrm{SS}$ in the region $\mathrm{A}$ is understandable, because the parameters $\eta_{\mathrm{t}}$ and $\eta_{\mathrm{r}}$ are large in this region. When passing from region $\mathrm{A}$ to region $\mathrm{B}$ with decreasing $\eta_{\mathrm{t}}$, the solutions change from SS to STS. On the other hand, from region A to region $\mathrm{C}$ with decreasing $\eta_{\mathrm{r}}$, the solutions change from SS to RS. These mode changes can be explained in terms of pitchfork bifurcations as we will see later in Fig. 3. From Fig. 2, we find that possible solutions which bifurcate from stationary solutions are travelling and rotating solutions. Hence, we consider the following restricted problems to capture these solutions.

The first restricted problem is the one neglecting the rotational motion. When taking into account the mirror symmetry of the elliptical shape, we can expect that the long-axis-directed 
travelling solution (LTS) potentially exists. To capture the solutions STS and LTS, we replace (16) with

$$
\left\{\begin{aligned}
m \frac{\mathrm{d}^{2} x_{\mathrm{c}}}{\mathrm{d} t^{2}} & =-\eta_{\mathrm{t}} \frac{\mathrm{d} x_{\mathrm{c}}}{\mathrm{d} t}+\mathbf{e}_{x} \cdot \int_{\partial \Omega_{\mathrm{c}}} \gamma(u) \boldsymbol{n} \mathrm{d} \ell, \\
y_{\mathrm{c}}(t) & \equiv y_{0},
\end{aligned}\right.
$$

and numerically solve the restricted problems (17)-(26) and (30) with $b / a=2$ for STS and $a / b=2$ for LTS, under the constraint $\theta_{\mathrm{c}}(t) \equiv 0$. Fig. 3(a) shows the bifurcation diagrams for these problems. As seen in Fig. 3(a), each STS primarily bifurcates from SS with a decrease in $\eta_{\mathrm{t}}$ and secondly LTS, which indicates that the STS are stable, while the LTS are unstable. The second restricted problem neglects the translational motion, in which we consider the system (16)-(26) under the constraint, $\boldsymbol{x}_{\mathrm{c}}(t) \equiv \boldsymbol{x}_{0}$. Fig. 3(b) shows the bifurcation diagram for this problem, in which a supercritical pitch-fork bifurcation occurs near $\eta_{\mathrm{r}}=0.0205$.

(a)

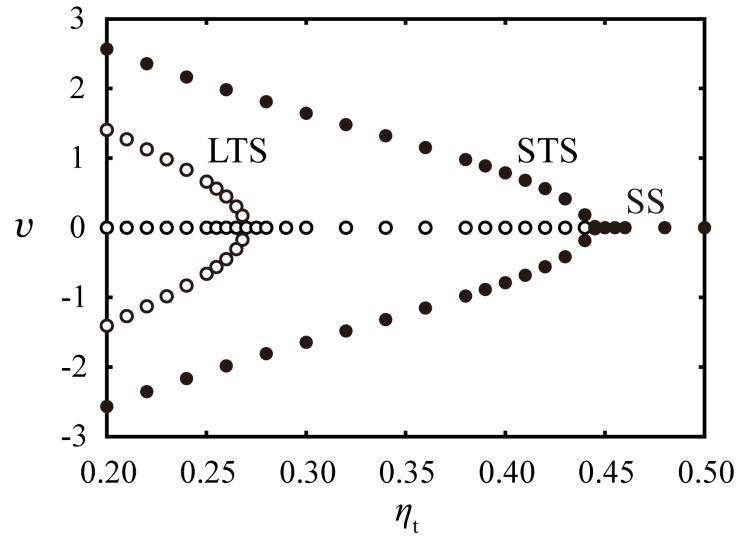

(b)

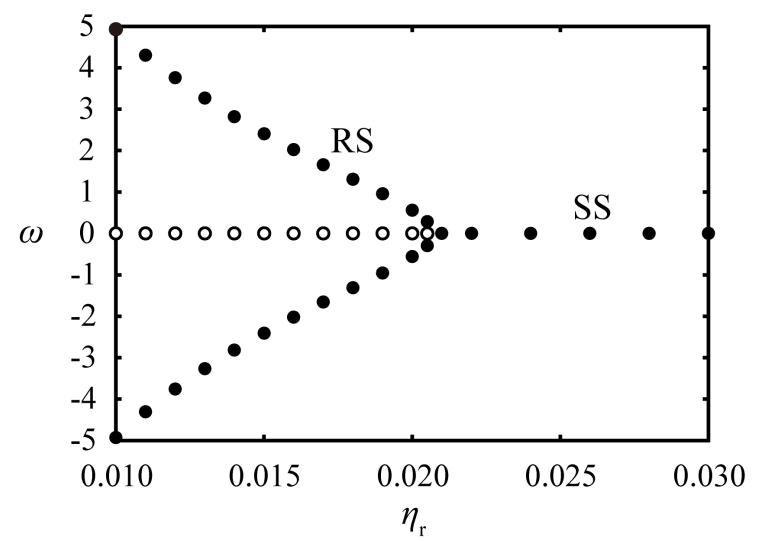

Fig. 3 (a) Bifurcation diagrams for the solutions SS, STS and LTS. The abscissa and ordinate denote $\eta_{\mathrm{t}}$ and $v$, respectively, where $v$ is the asymptotic velocity numerically computed for large $t$. (b) Bifurcation diagram for the solutions SS and RS. The abscissa and ordinate denote $\eta_{\mathrm{r}}$ and $\omega$, respectively, where $\omega$ is the asymptotic angular velocity numerically computed for large $t$. In (a) and (b), the filled circle denotes the stable solution, while the empty circle denotes the unstable one. All parameters are the same as those in Fig. 2.

From Figs. 2 and 3, we can predict that camphor particles with elliptical shape tend to move in the short-axis direction at least in the case that $\eta_{\mathrm{r}} \gg 0$. To confirm this result for different ellipticity, we numerically compute the bifurcation points of the LTS (symbolized by $\eta_{\text {LTS }}$ ) and those of STS (symbolized by $\eta_{\mathrm{STS}}$ ) based on the restricted problem (18)-(26) and (30), as a function of the parameter $a$ subject to the constraint that the area of the camphor particle is kept constant at $\pi r_{0}^{2}$, where $r_{0}$ is a positive constant. Fig. 4 shows the bifurcation curve on which the bifurcation points of the numerical solutions LTS and STS are plotted. In the figure, one can see that $\eta_{\text {STS }}$ 
is greater than $\eta_{\text {LTS }}$ for any value of $a\left(\neq r_{0}\right)$, and that $\eta_{\text {STS }}$ increases with an increase in $b$ near the point at $a=b=r_{0}$. This means that the elliptical deformation of a circular camphor disk can drive its short-axis-directed motion.

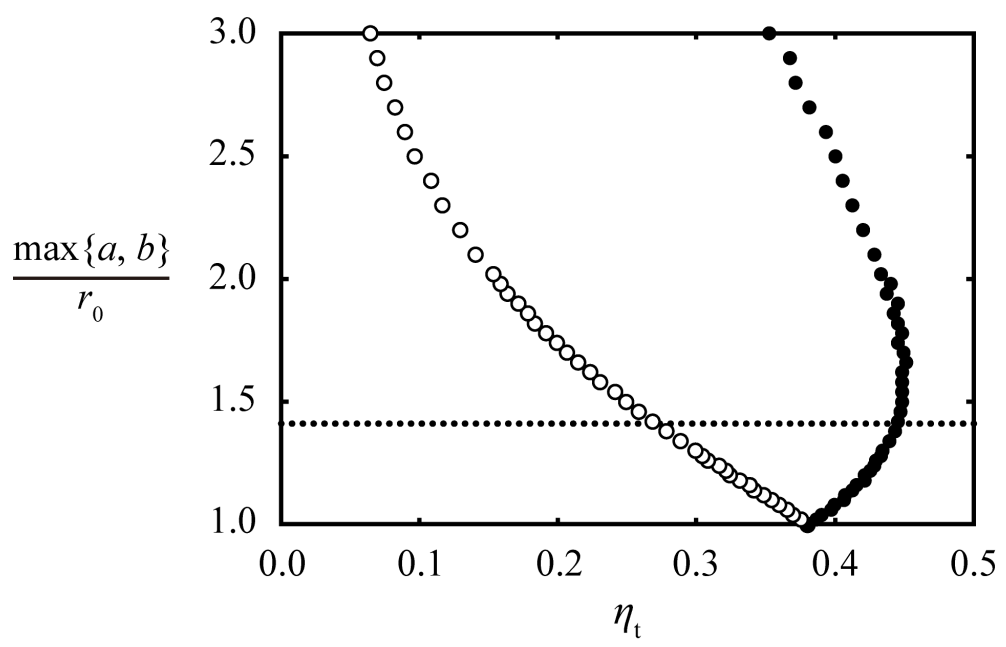

Fig. 4 Bifurcation curves obtained from the numerical computations with the restricted problems (17)-(26) and (30) in which the area of the camphor particle is kept constant at $\pi r_{0}^{2}$. The empty and filled circles respectively denote $\left(\eta_{\mathrm{LTS}}, a / r_{0}\right)$ and $\left(\eta_{\mathrm{STS}}, b / r_{0}\right)$, which are the bifurcation points of the long- and short-axis-directed travelling solutions. The horizontal dotted line represents $\max \{a, b\} / r_{0}=\sqrt{2}$, which is the same as that in Fig. 2 .

\section{Analytical results}

When a camphor particle has an exact circular shape, $f$ in (18) has the circular symmetry. Hence, (16) and (18) are independent of $\theta_{c}$. Thus, (17) is separated from the system and can be neglected. In this case, the system, (15)-(25), becomes essentially the same as the existing model [19]. Generalized model, in which $\Omega_{\mathrm{c}}$ is defined as a disk with $k$-mode deformation, enables us to investigate the contribution of the particle shape to the motion. As a special case of $k=2$, the camphor particle has an elliptic shape whose magnitude of deformation from a circular shape is infinitesimally small. Hereinafter, we call this camphor particle an "elliptic camphor disk".

In Section 4.1, we investigate bifurcations from stationary to travelling solutions without regard to rotation, which offers helpful suggestions on the direction of motion of the elliptic camphor disk in the experiment. In Section 4.2, we also investigate bifurcation from stationary to rotating solution without regard to translation, which provides us with better understanding on the relationship between elliptic deformation and rotation.

\subsection{Analysis I: translation of an elliptic camphor disk}

Based on (1), we define the shape of the elliptic camphor disk as follows:

$$
\partial \Omega_{\mathrm{c}}\left(\boldsymbol{x}_{\mathrm{c}}\right)=\left\{(x, y)=\left(x_{\mathrm{c}}, y_{\mathrm{c}}\right)+r_{0}(1+\varepsilon \cos 2 \theta)(\cos \theta, \sin \theta) \mid 0 \leq \theta<2 \pi\right\}
$$


where $\varepsilon$ is a parameter for the elliptic deformation from a circle with radius $r_{0}$. We take $|\varepsilon| \ll 1$, then (31) becomes a first approximation of (26) by letting $a=r_{0}(1+\varepsilon)$ and $b=r_{0}(1-\varepsilon)$.

Let us assume that the elliptic camphor disk is moving along the $x$ axis at a constant velocity $v \mathbf{e}_{x}$, where $\mathbf{e}_{x}$ is the unit vector in the $x$-axis direction. Note that if $\varepsilon>0$, then the camphor disk moves in the long-axis direction, while if $\varepsilon<0$, it moves in the short-axis direction (see Fig. 5). In both cases, it is expected that the profile of $u$ has the mirror symmetry with respect to the longor short-axis direction, and hence the torque is cancelled:

$$
\int_{\partial \Omega_{\mathrm{c}}} \gamma(u) \boldsymbol{p} \times \boldsymbol{n} \mathrm{d} \ell=0 .
$$

Thus, under the conditions as in Fig. 5, we can neglect the equation of rotation (17). The above constraint can be proved through the following analysis, but the details are not given here for the sake of brevity.
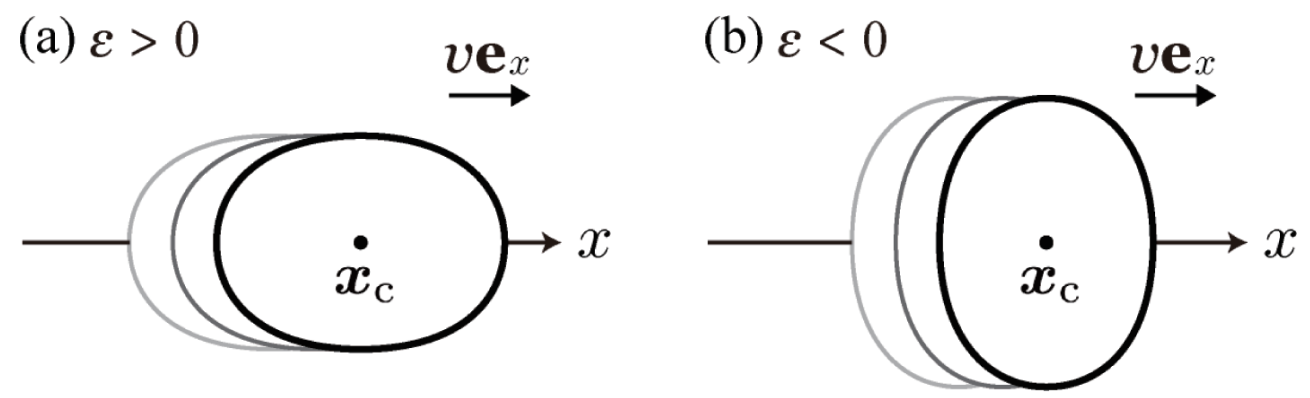

Fig. 5 Schematic representation for the assumption.

To view the system from a co-moving frame, we introduce $\boldsymbol{z}(=(z, y)), \boldsymbol{z}_{\mathrm{c}}\left(=\left(z_{\mathrm{c}}, y_{\mathrm{c}}\right)\right)$ and $U$ such that

$$
\boldsymbol{z}(t)=(x-v t, y), \quad \boldsymbol{z}_{\mathrm{c}}(t)=\left(x_{\mathrm{c}}-v t, y_{\mathrm{c}}\right), \quad U(\boldsymbol{z})=u(t, \boldsymbol{x}) .
$$

Then, (16) and (18) become

$$
\begin{gathered}
\mathbf{0}=-\eta_{\mathrm{t}} v \mathbf{e}_{z}+\int_{\partial \Omega_{\mathrm{c}}} \gamma(U) \boldsymbol{n} \mathrm{d} \ell, \\
-v \frac{\partial U}{\partial z}=\Delta U-U+f\left(\boldsymbol{z}, \boldsymbol{z}_{\mathrm{c}}\right),
\end{gathered}
$$

where

$$
f\left(\boldsymbol{z}, \boldsymbol{z}_{\mathrm{c}}\right)= \begin{cases}1, & \boldsymbol{z} \in \bar{\Omega}_{\mathrm{c}} \\ 0, & \boldsymbol{z} \in \Omega \backslash \bar{\Omega}_{\mathrm{c}}\end{cases}
$$


and (24) and (25) become

$$
\begin{gathered}
\lim _{|\boldsymbol{z}| \rightarrow \infty} U(\boldsymbol{z})=0, \\
U(\cdot) \in C^{1}(\Omega) .
\end{gathered}
$$

We can set $\boldsymbol{z}_{\mathrm{c}}(t) \equiv \mathbf{0}$ without loss of generality.

Next, we introduce the polar coordinates $(r, \theta)$ such that

$$
(z, y)=(r \cos \theta, r \sin \theta), \quad U(r, \theta):=U(\boldsymbol{z})
$$

after which (31)-(36) become as follows:

$$
\begin{gathered}
\partial \Omega_{\mathrm{c}}(r, \theta)=\left\{(r, \theta) \in \Omega \mid r=r_{0}(1+\varepsilon \cos 2 \theta), 0 \leq \theta<2 \pi\right\} \\
\mathbf{0}=-\eta_{\mathrm{t}} v \mathbf{e}_{z}+\int_{0}^{2 \pi} \gamma\left(U\left(r_{0}(1+\varepsilon \cos 2 \theta), \theta\right)\right) \boldsymbol{n}(\theta) r_{0}\left(1+\varepsilon \cos 2 \theta+O\left(\varepsilon^{2}\right)\right) \mathrm{d} \theta \\
-v\left(\cos \theta \frac{\partial U}{\partial r}-\frac{\sin \theta}{r} \frac{\partial U}{\partial \theta}\right)=\frac{\partial^{2} U}{\partial r^{2}}+\frac{1}{r} \frac{\partial U}{\partial r}+\frac{1}{r^{2}} \frac{\partial^{2} U}{\partial \theta^{2}}-U+f(r, \theta), \\
f(r, \theta)= \begin{cases}1, & r \leq r_{0}(1+\varepsilon \cos 2 \theta), \\
0, & r>r_{0}(1+\varepsilon \cos 2 \theta), \\
\lim _{r \rightarrow \infty} U(r, \theta)=0, \\
U(\cdot, \theta) \in C^{1}((0, \infty)),\end{cases}
\end{gathered}
$$

where

$$
\boldsymbol{n}(\theta)=(\cos \theta-2 \varepsilon \sin \theta \sin 2 \theta, \sin \theta+2 \varepsilon \cos \theta \sin 2 \theta)+\boldsymbol{O}\left(\varepsilon^{2}\right) .
$$

From (37)-(41), we can calculate the bifurcation point of the travelling solution by means of perturbation method (see Appendix A in detail). Let $U(r, \theta ; \varepsilon, v)$ be a function satisfying (39)-(41) and consider the expansion of $U(r, \theta ; \varepsilon, v)$ with respect to an infinitesimally small $\varepsilon$ and $v$ as follows

$$
U(r, \theta ; \varepsilon, v)=\sum_{n=0}^{\infty}\left(U_{n}(r, \theta)+\varepsilon \tilde{U}_{n}(r, \theta)\right) v^{n}+O\left(\varepsilon^{2}\right)
$$

where $U_{n}$ and $\tilde{U}_{n}(n=0,1,2, \cdots)$ are the functions satisfying (40) and (41). Substituting (42) into $U$ in (39), we successively obtain $U_{n}$ and $\tilde{U}_{n}(n=0,1,2, \cdots)$. Substituting (42) into $U$ in (38) yields

$$
\begin{aligned}
\eta_{\mathrm{t}} v & =\mathbf{e}_{z} \cdot \int_{0}^{2 \pi} \gamma\left(U\left(r_{0}(1+\varepsilon \cos 2 \theta), \theta ; \varepsilon, v\right)\right) \boldsymbol{n}(\theta) r_{0}(1+\varepsilon \cos 2 \theta) \mathrm{d} \theta+O\left(\varepsilon^{2}\right) \\
& =\left(k_{1}+\varepsilon \tilde{k}_{1}\right) v+\left(k_{3}+\varepsilon \tilde{k}_{3}\right) v^{3}+O\left(\varepsilon^{2}\right)+O\left(v^{5}\right)
\end{aligned}
$$


where $k_{1}, \tilde{k}_{1}, k_{3}$ and $\tilde{k}_{3}$ are the constants determined by the parameters. For example, $k_{1}$ and $\tilde{k}_{1}$ are given as follows:

$$
\begin{aligned}
& k_{1}=-\frac{\pi r_{0}^{4}}{4} \gamma^{\prime}\left(U_{00}\right)\left(I_{0} K_{0}-I_{2} K_{2}\right), \\
& \tilde{k}_{1}=\frac{\pi r_{0}^{4}}{2} \gamma^{\prime}\left(U_{00}\right)\left(I_{1} K_{1}-I_{2} K_{2}\right)-\frac{\pi r_{0}^{7}}{32} \gamma^{\prime \prime}\left(U_{00}\right)\left(I_{0} K_{0}-I_{2} K_{2}\right)\left(I_{1} K_{1}-I_{2} K_{2}-\frac{4}{r_{0}} I_{1} K_{1}\right),
\end{aligned}
$$

where we define $I_{n}=\mathcal{I}_{n}\left(r_{0}\right), K_{n}=\mathcal{K}_{n}\left(r_{0}\right)$ and $U_{00}=r_{0} I_{1} K_{0}$. Here, $k_{1}$ is shown to be positive from the following inequality [32]:

$$
\mathcal{I}_{m}(r) \mathcal{K}_{m}(r)-\mathcal{I}_{n}(r) \mathcal{K}_{n}(r)>0 \quad(m<n) .
$$

Hence, (43) shows that a pitch-fork bifurcation occurs at $\eta_{\mathrm{t}}=k_{1}+\varepsilon \tilde{k}_{1}$ and that the velocity of the travelling solution near the bifurcation point is given by

$$
v \approx \pm \sqrt{\frac{\eta_{\mathrm{t}}-\left(k_{1}+\varepsilon \tilde{k}_{1}\right)}{k_{3}+\varepsilon \tilde{k}_{3}}} .
$$

Furthermore, one can see that for small $r_{0}$ the sign of $\tilde{k}_{1}$ is mainly governed by the first term of the right-hand side of (45), which is negative. In fact, with the same parameters as those in Fig. 2,

$$
k_{1} \approx 0.380288, \quad \tilde{k}_{1} \approx-0.266656 .
$$

Considering that the $x$ axis corresponds to the long- and short-axis direction when $\varepsilon$ is positive and negative, respectively, and that $\tilde{k}_{1}$ is negative, we find that the bifurcation point of the longaxis-directed travelling solution is smaller than that of the short-axis-directed one. This means that the elliptic camphor disk is easier to move in the short-axis direction within the neighborhood of the bifurcation point. Fig. 6 shows the bifurcation curve obtained from (43) and the numerical computation, on which $\eta_{\mathrm{t}}$ and $\varepsilon$ at the bifurcation point are plotted. In the figure, the gray line and empty circles are for $\varepsilon>0$, while the black line and filled circles are for $\varepsilon<0$. This shows that the travelling solutions bifurcate from the stationary solution for the circular camphor disk, whose bifurcation point changes with $O(\varepsilon)$.

Finally, we examine the bifurcation type, super- or sub-critical, of the travelling solution for a circular camphor disk. Set $\varepsilon=0$ in (43), then we obtain

$$
\eta_{\mathrm{t}} v=k_{1} v+k_{3} v^{3}+O\left(v^{5}\right),
$$

where

$$
\begin{aligned}
k_{3} & =\frac{\pi r_{0}^{6}}{32} \gamma^{\prime}\left(U_{00}\right)\left(-I_{0} K_{0}+\frac{2}{r_{0}^{2}} I_{1} K_{1}+I_{2} K_{2}\right) \\
& +\frac{\pi r_{0}^{8}}{512} \gamma^{\prime \prime}\left(U_{00}\right)\left(-I_{0} K_{0}+I_{2} K_{2}\right)\left\{10 I_{0} K_{0}-\left(\frac{16}{r_{0}^{2}}-1\right) I_{1} K_{1}-10 I_{2} K_{2}+I_{3} K_{3}\right\} \\
& +\frac{\pi r_{0}^{10}}{512} \gamma^{(3)}\left(U_{00}\right)\left(-I_{0} K_{0}+I_{2} K_{2}\right)^{2} .
\end{aligned}
$$




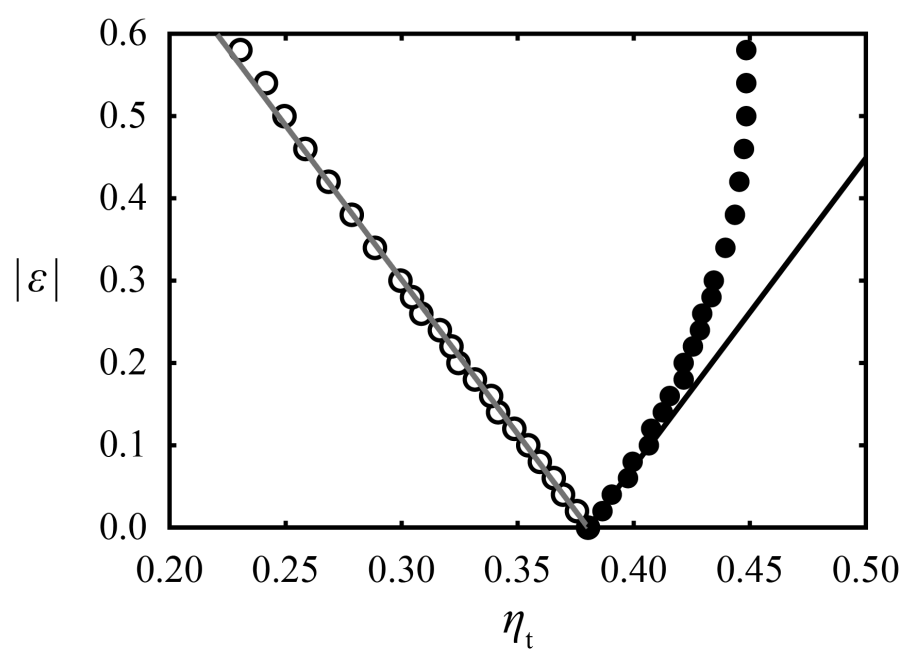

Fig. 6 Bifurcation curves obtained from analytical and numerical calculations. The gray and black lines are obtained from (43) while the empty and filled circles are from the numerical computation performed by the same manners with that of Fig. 4. The gray line and empty circles are for $\varepsilon>0$, while the black line and filled circles are for $\varepsilon<0$.

It is noteworthy that if $\gamma$ is defined as a linear function, i.e., $\gamma^{\prime \prime}\left(U_{00}\right)$ and $\gamma^{(3)}\left(U_{00}\right)$ are zero, then $k_{3}$ has a negative value [31]. However, since $\gamma$ is the nonlinear function (19), the sign of $k_{3}$ can depend on the parameters of the model. Fig. 7 shows the nullcline of $k_{3}\left(r_{0}, \beta\right)=0$ by taking $r_{0}$ and $\beta$ as control parameters, where $r_{0}$ is given in (31) and $\beta$ in (19). As seen in the figure, one can see that $k_{3}$ is positive for small $\beta$, which induces a subcritical pitch-fork bifurcation. Experimental verification of this prediction is an interesting problem, which remains for future work.

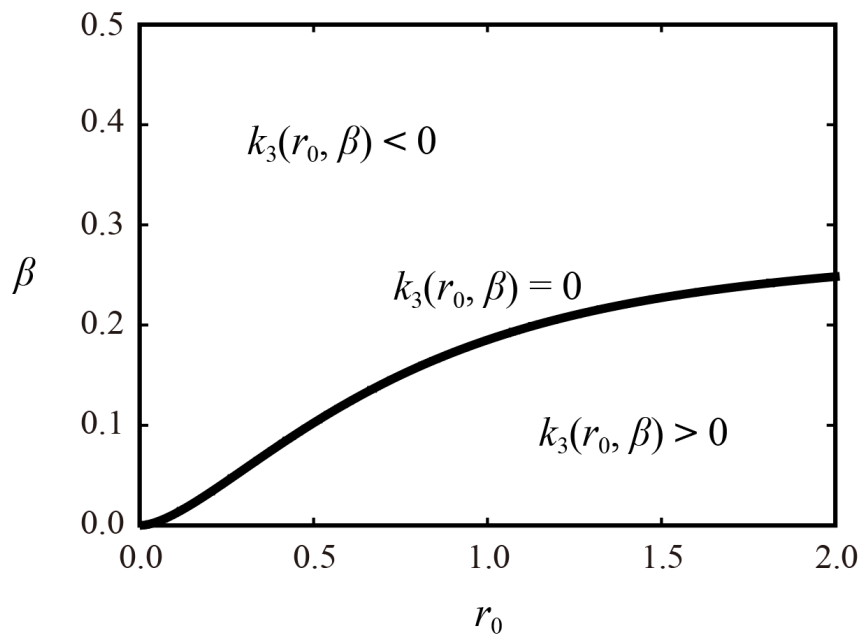

Fig. 7 The nullcline of $k_{3}\left(r_{0}, \beta\right)=0$, where $\gamma_{0}=2.5, n=2$. 


\subsection{Analysis II: rotation of an elliptic camphor disk}

Based on (1), we define the shape of the elliptic camphor disk as follows:

$$
\partial \Omega_{\mathrm{c}}\left(\theta_{\mathrm{c}}\right)=\left\{(x, y)=r_{0}(1+\varepsilon \cos 2 \theta)(\cos \theta, \sin \theta) R\left(-\theta_{\mathrm{c}}\right) \mid 0 \leq \theta<2 \pi\right\},
$$

where $\varepsilon$ is an infinitesimal parameter for the elliptic deformation from a circle with a radius $r_{0}$.

Let us assume that the elliptic camphor disk is rotating at a constant angular velocity $\omega$ with its center at the origin (see Fig. 8). In this case, it is expected that the profile of $u$ has the $\pi$-rotational symmetry with respect to $\boldsymbol{x}_{\mathrm{c}}$, and hence the driving force for translation is cancelled:

$$
\int_{\partial \Omega_{\mathrm{c}}} \gamma(u) \boldsymbol{n} \mathrm{d} \ell=\mathbf{0}
$$

Thus, under the condition as in Fig. 8, we can neglect the equation of translation (16). The above constraint can be proved through the following analysis, but the details are not given here for the sake of brevity.

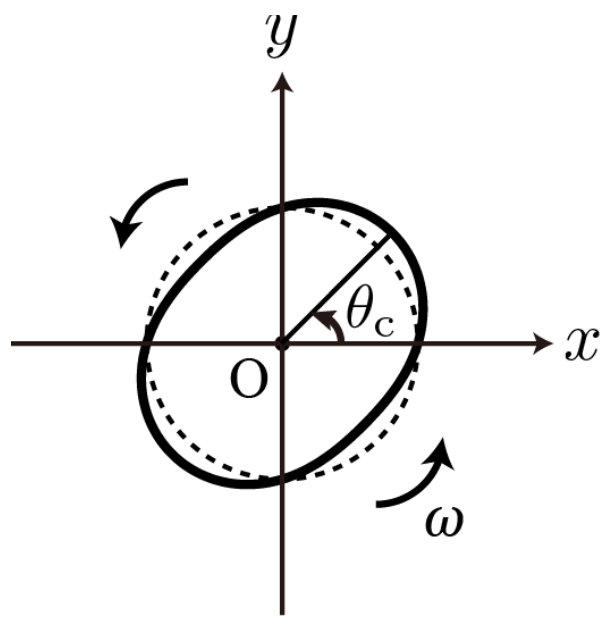

Fig. 8 Schematic representation for the assumption. The thick solid line represents the elliptic camphor disk with $|\varepsilon| \ll 1$, while the dashed line represents the circular disk.

To view the system from a co-rotating frame, we introduce $\boldsymbol{\xi}\left(=\left(\xi_{1}, \xi_{2}\right)\right)$, $\phi_{\mathrm{c}}$ and $V$ such that

$$
\boldsymbol{\xi}(t)=(x, y) R(\omega t), \quad \phi_{\mathrm{c}}(t)=\theta_{\mathrm{c}}-\omega t, \quad V(\boldsymbol{\xi})=u(t, \boldsymbol{x}) .
$$

Then, (17) and (18) become

$$
\begin{gathered}
0=-\eta_{\mathrm{r}} \omega+\int_{\partial \Omega_{\mathrm{c}}} \gamma(V) \boldsymbol{p} \times \boldsymbol{n} \mathrm{d} \ell, \\
\omega\left(\frac{\partial V}{\partial z} \xi_{2}-\frac{\partial V}{\partial w} \xi_{1}\right)=\Delta V-V+f\left(\boldsymbol{\xi}, \phi_{\mathrm{c}}\right),
\end{gathered}
$$


where

$$
f\left(\boldsymbol{\xi}, \phi_{\mathrm{c}}\right)= \begin{cases}1, & \boldsymbol{\xi} \in \bar{\Omega}_{\mathrm{c}} \\ 0, & \boldsymbol{\xi} \in \Omega \backslash \bar{\Omega}_{\mathrm{c}}\end{cases}
$$

and (24) and (25) become

$$
\begin{gathered}
\lim _{|\boldsymbol{\xi}| \rightarrow \infty} V(\boldsymbol{\xi})=0, \\
V(\cdot) \in C^{1}(\Omega) .
\end{gathered}
$$

We can set $\phi_{\mathrm{c}}(t) \equiv 0$ without loss of generality.

Next, we introduce the polar coordinates $(r, \theta)$ such that

$$
\left(\xi_{1}, \xi_{2}\right)=(r \cos \theta, r \sin \theta), \quad U(r, \theta):=U(\boldsymbol{\xi}),
$$

after which (47)-(52) become

$$
\begin{gathered}
\partial \Omega_{\mathrm{c}}(r, \theta)=\left\{(r, \theta) \in \Omega \mid r=r_{0}(1+\varepsilon \cos 2 \theta), 0 \leq \theta<2 \pi\right\}, \\
0=-\eta_{\mathrm{r}} \omega+\int_{0}^{2 \pi} \gamma\left(V\left(r_{0}(1+\varepsilon \cos 2 \theta), \theta\right)\right) \boldsymbol{p}(\theta) \times \boldsymbol{n}(\theta) r_{0}\left(1+\varepsilon \cos 2 \theta+O\left(\varepsilon^{2}\right)\right) \mathrm{d} \theta, \\
-\omega \frac{\partial V}{\partial \theta}=\frac{\partial^{2} V}{\partial r^{2}}+\frac{1}{r} \frac{\partial V}{\partial r}+\frac{1}{r^{2}} \frac{\partial^{2} V}{\partial \theta^{2}}-V+f(r, \theta), \\
f(r, \theta)= \begin{cases}1, & r \leq r_{0}(1+\varepsilon \cos 2 \theta), \\
0, & r>r_{0}(1+\varepsilon \cos 2 \theta), \\
\lim _{r \rightarrow \infty} V(r, \theta)=0, \\
V(\cdot, \theta) \in C^{1}((0, \infty)),\end{cases}
\end{gathered}
$$

where

$$
\boldsymbol{p}(\theta) \times \boldsymbol{n}(\theta)=\left(2 r_{0} \sin 2 \theta\right) \varepsilon+O\left(\varepsilon^{3}\right) .
$$

From (53)-(58), we can calculate the bifurcation point of the rotating solution by means of perturbation method (see Appendix B in detail). Let $V(r, \theta ; \varepsilon, \omega)$ be a function satisfying (55)(58) and consider the expansion of $V(r, \theta ; \varepsilon, \omega)$ with respect to an infinitesimally small $\varepsilon$ and $\omega$ as follows:

$$
V(r, \theta ; \varepsilon, \omega)=\sum_{n=0}^{\infty}\left(V_{n}(r, \theta)+\varepsilon \tilde{V}_{n}(r, \theta)\right) \omega^{n}+O\left(\varepsilon^{2}\right)
$$


where $V_{n}$ and $\tilde{V}_{n}(n=0,1,2, \cdots)$ are the functions satisfying (57) and (58). Substituting (59) into $V$ in (55), we successively obtain $V_{n}$ and $\tilde{V}_{n}(n=0,1,2, \cdots)$. Substituting (59) into $V$ in (54) yields

$$
\begin{aligned}
\eta_{\mathrm{r}} \omega & =\int_{0}^{2 \pi} \gamma\left(V\left(r_{0}(1+\varepsilon \cos 2 \theta), \theta ; \varepsilon, \omega\right)\right) \boldsymbol{p}(\theta) \times \boldsymbol{n}(\theta) r_{0}(1+\varepsilon \cos 2 \theta) \mathrm{d} \theta+O\left(\varepsilon^{3}\right), \\
& =\varepsilon^{2}\left(\tilde{l}_{1} \omega+\tilde{l}_{3} \omega^{3}\right)+O\left(\varepsilon^{3}\right)+O\left(\omega^{5}\right) .
\end{aligned}
$$

The constants $\tilde{l}_{1}$ and $\tilde{l}_{3}$ are given as follows:

$$
\begin{aligned}
& \tilde{l}_{1}=\frac{\pi r_{0}^{6}}{2} \gamma^{\prime}\left(V_{00}\right)\left(-I_{1} K_{1}+I_{3} K_{3}\right), \\
& \tilde{l}_{3}=\frac{\pi r_{0}^{8}}{18} \gamma^{\prime}\left(V_{00}\right)\left(3 I_{1} K_{1}-2 I_{2} K_{2}-3 I_{3} K_{3}+2 I_{4} K_{4}\right),
\end{aligned}
$$

where we define $I_{n}=\mathcal{I}_{n}\left(r_{0}\right), K_{n}=\mathcal{K}_{n}\left(r_{0}\right)$ and $V_{00}=r_{0} I_{1} K_{0}$. Here, $\tilde{l}_{1}$ is shown to be positive while $\tilde{l}_{3}$ be negative from (46) and the following inequality [32]:

$$
2 \mathcal{I}_{n}(r) \mathcal{K}_{n}(r) \leq \mathcal{I}_{n-1}(r) \mathcal{K}_{n-1}(r)+\mathcal{I}_{n+1}(r) \mathcal{K}_{n+1}(r)
$$

(Taking $\psi_{n}:=I_{n} K_{n}$ in $(62)$, we see that $3 \psi_{1}-2 \psi_{2}-3 \psi_{3}+2 \psi_{4} \geq 2 \psi_{1}-4 \psi_{3}+2 \psi_{4} \geq 2\left(\psi_{1}-\psi_{2}\right)>0$.) Hence, (60) shows that a supercritical pitch-fork bifurcation occurs at $\eta_{\mathrm{r}}=\varepsilon^{2} \tilde{l}_{1}$ and that the angular velocity of the rotating solution near the bifurcation point is given by

$$
\omega \approx \pm \frac{1}{\varepsilon} \sqrt{\frac{\eta_{\mathrm{r}}-\varepsilon^{2} \tilde{l}_{1}}{\tilde{l}_{3}}} \quad(|\varepsilon| \neq 0) .
$$

Fig. 9 shows the bifurcation curve on which $\eta_{\mathrm{r}}$ and $\varepsilon$ at the bifurcation point are plotted. In the figure, the solid line is obtained from (60) and the filled circles from numerical computation. This shows that the rotating solution bifurcates from the stationary solution for the circular camphor disk, whose bifurcation point changes with $O\left(\varepsilon^{2}\right)$.

\section{Experiment}

\subsection{Experimental method}

In this section, we examine the motion of an elliptic camphor particle on water experimentally. We used (+)-Camphor from Wako Pure Chemical Industries Co., Ltd. (Osaka, Japan) and purified water from Matsuba Chemical Co., Ltd. (Osaka, Japan). The elliptic camphor particle was prepared by putting camphor powder into an elliptical template (No. E201N, Sanko, Japan). The upper surface of the camphor particle was colored with black ink for easier visualization. Then, we prepared a rectangular water bath (size: ca. 32 by $23 \mathrm{~cm}$ ), in which we poured 1000-mL of purified water. The depth of the water in the bath was approximately $15 \mathrm{~mm}$. After placing the elliptic camphor particle (size: ca. 13.5 by $6.5 \mathrm{~mm}$, thickness: ca. $1 \mathrm{~mm}$ ) on the water, we monitored its motion with a digital video camera (Sony HDR-XR520V) from above. All measurements were carried out at least three times at room temperature $\left(\right.$ ca. $23{ }^{\circ} \mathrm{C}$ ). The images were then analyzed with image processing software (ImageJ, National Institutes of Health, USA). 


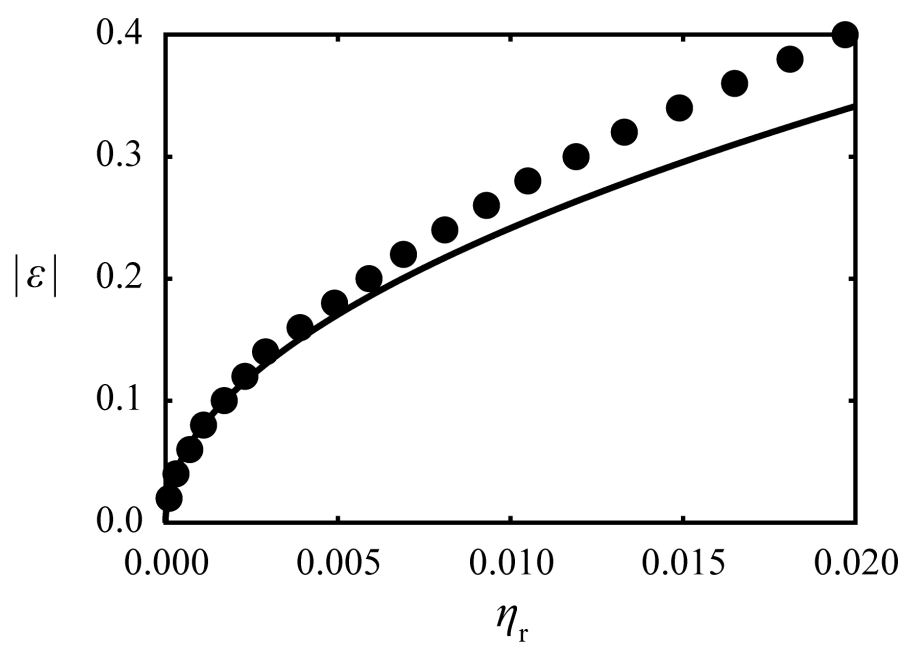

Fig. 9 Bifurcation curves obtained from the analytical and numerical calculations. The solid line is obtained from (60) and the filled circles from the numerical computation with the restricted problem neglecting the translational motion, in which the area of the camphor particle is kept constant at $\pi r_{0}^{2}$.

\subsection{Experimental results}

Fig. 10 shows the experimental results of the motion of the elliptic camphor particle on water. In Fig. 10(a), one can see that the camphor particle moves mainly in the short-axis direction. To clarify the tendency of the motion in the short-axis direction, we examined the angle profile $(\chi(t), \phi(t))$ throughout a 60 second interval, where $\chi(t)$ is the angle between the $x$-axis and the directional vector in the short-axis of the elliptic camphor particle, and $\phi(t)$ is the angle between the $x$-axis and the velocity vector (see Figs. 10(b) and (c)). From Fig. 10(b), we found that $\phi(t) \approx \chi(t)$, which means that the camphor particle moves mainly in the short-axis direction. This experimental result is consistent with our theoretical one.

\section{Conclusion}

Our numerical and analytical results showed that travelling and rotating solutions bifurcate from stationary solutions via pitch-fork bifurcations, and that the stable travelling solution is directed in the short axis. This result may be intuitively explained as follows: at least there are two perspectives considered:

(i) Projected length of an ellipse on the long axis is larger than that on the short axis, which induces the greater driving force in the short-axis direction.

(ii) The difference of $u$ between both sides of the camphor in the long-axis direction is greater than that in the short-axis direction, which induces the greater driving force in the long-axis direction. 


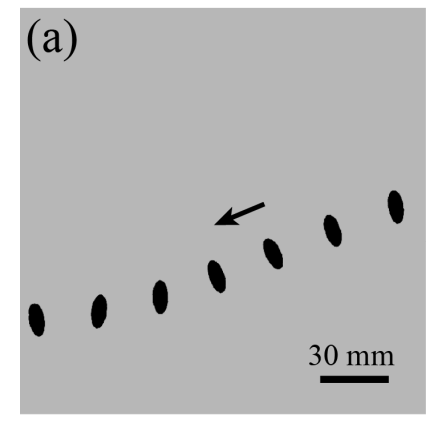

(b)

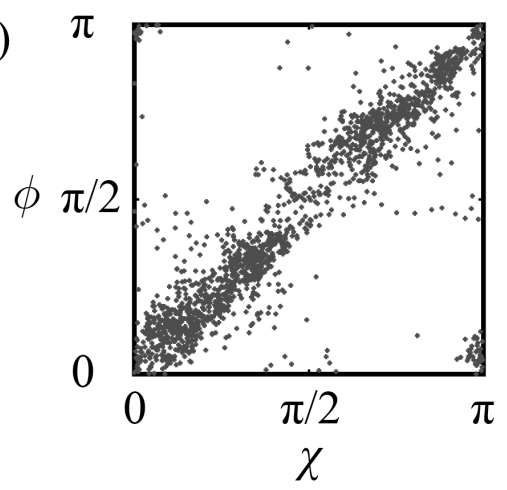

(c)

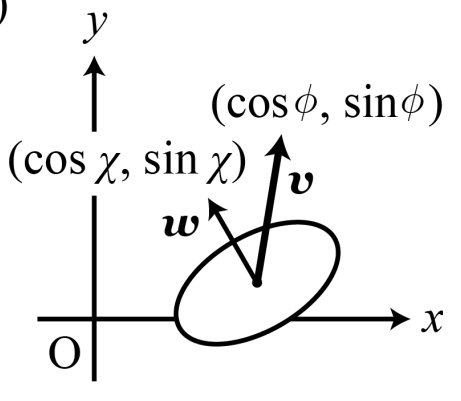

Fig. 10 Experimental results on the motion of an elliptic camphor particle on water [31]. (a) Superposed snapshots, each of which is taken every $1 / 6$ seconds. It can be seen that the camphor particle moves from right to left with an almost constant velocity. (b) Correlation between the angles $\chi$ and $\phi$. (c) Definition of $\chi$ and $\phi$, which are the angles of $\boldsymbol{v}$ and $\boldsymbol{w}$ from the $x$ axis, respectively. Here, $\boldsymbol{v}$ is the velocity vector and $\boldsymbol{w}$ is the directional vector in the short-axis direction of the elliptic camphor particle. The plotted data (b) were obtained from the experimental movie which was taken for 60 seconds.

Although (i) and (ii) conflicts with each other, the contribution of (i) is more effective than that of (ii) in our present camphor-water system, which results in motions in the short-axis direction.

Analytical results clarified that the bifurcation point of the long- and short-axis-directed travelling solutions change with $O(\varepsilon)$ while that of the rotating solution changes with $O\left(\varepsilon^{2}\right)$ for small $\varepsilon$. Thus, we found that the elliptic camphor disk is easier to exhibit translation than rotation, at least within the infinitesimally small $\varepsilon$. The above results were confirmed by a camphor-water system experiment, which showed that elliptic camphor particles tend to move straightly in the short-axis direction.

Applying the perturbation method as shown above, we can also calculate the bifurcation points of the travelling and rotating solutions for the camphor disk with $k$-mode deformation. As a result, we can suggest that the deformation of $k$-mode for $k \geq 3$ does not affect the translational motion as far as the deformation of first order of $\varepsilon$ (infinitesimally small parameter for deformation) is considered. Of course, by considering the second order of $\varepsilon$, every mode can affect the translational motion through the mode coupling, which becomes the calculation more complicated. In contrast, every mode for $k \geq 3$ affects the rotational motion in the same way as that for $k=2$ mode; the rotation is induced in the order of $\varepsilon^{2}$. It is noted that the coefficient for the torque is larger as $k$ is larger, i.e., large-mode deformation strongly drives rotational motion.

The present study provides us with the following future work. First, stability analysis of the elliptic camphor disk travelling solution is necessary for a more rigorous discussion. According to the [19], the stable stationary solution of circular camphor disks becomes unstable with a decrease in the friction parameter for translation. We would like to apply this theory to our present system to show that the solution can converge to the short-axis-directed travelling solution. Second, to make the mathematical model more realistic, we should consider the inertia resistance as well as the hydrodynamic effect such as Marangoni convection. Finally, clarifying the global bifurcation 
structure hidden in Fig. 2 remains an interesting problem. Although the details are not given in the present paper, we numerically revealed that for small $\eta_{\mathrm{r}}$ the short-axis-directed travelling solution bifurcates from the stationary solution and the travelling-rotating solution bifurcates from the rotating solution. To tackle this problem analytically, we must consider large elliptical deformations of the particle shape, to which we hope that we can apply elliptic coordinates and the theory of Mathieu functions.

\section{Acknowledgements}

The authors thank Dr. Y. Kobayashi (Hokkaido University) for helpful discussion. This work was supported in part by Grants-in-Aid for Young Scientists (B) given to H. K. (No. 24740256) and Scientific Research (B) given to M. N. (No. 21340023). 


\section{Appendix A. Perturbation method for the travelling solution}

To obtain $U_{n}$ and $\tilde{U}_{n}(n=0,1,2, \cdots)$ in (42), we first consider the following expansion of $U(r, \theta ; \varepsilon, v)$ :

$$
U(r, \theta ; \varepsilon, v)=\sum_{n=0}^{\infty} U_{n}(r, \theta ; \varepsilon) v^{n}
$$

where $U_{n}(r, \theta ; \varepsilon)(n=0,1,2, \cdots)$ are the functions satisfying the following equation for $n=0$ :

$$
\begin{gathered}
0=\frac{\partial^{2} U_{n}}{\partial r^{2}}+\frac{1}{r} \frac{\partial U_{n}}{\partial r}+\frac{1}{r^{2}} \frac{\partial^{2} U_{n}}{\partial \theta^{2}}-U_{n}+f(r, \theta), \\
f(r, \theta)= \begin{cases}1, & r \leq r_{0}(1+\varepsilon \cos 2 \theta), \\
0, & r>r_{0}(1+\varepsilon \cos 2 \theta),\end{cases}
\end{gathered}
$$

and for $n=1,2, \cdots$,

$$
-\left(\cos \theta \frac{\partial U_{n-1}}{\partial r}-\frac{\sin \theta}{r} \frac{\partial U_{n-1}}{\partial \theta}\right)=\frac{\partial^{2} U_{n}}{\partial r^{2}}+\frac{1}{r} \frac{\partial U_{n}}{\partial r}+\frac{1}{r^{2}} \frac{\partial^{2} U_{n}}{\partial \theta^{2}}-U_{n}
$$

as well as the following conditions:

$$
\begin{gathered}
\lim _{r \rightarrow \infty} U_{n}(r, \theta)=0 \\
\lim _{r \rightarrow+0} U_{n}(r, \theta)<\infty, \quad \lim _{r \rightarrow+0} \frac{\partial U_{n}}{\partial r}(r, \theta)=0 \\
U_{n}^{(\mathrm{i})}\left(r_{0}, \theta\right)+\left.\varepsilon \frac{\partial U_{n}^{(\mathrm{i})}}{\partial r}\right|_{r=r_{0}} r_{0} \cos 2 \theta=U_{n}^{(\mathrm{o})}\left(r_{0}, \theta\right)+\left.\varepsilon \frac{\partial U_{n}^{(\mathrm{o})}}{\partial r}\right|_{r=r_{0}} r_{0} \cos 2 \theta, \\
\left.\frac{\partial U_{n}^{(\mathrm{i})}}{\partial r}\right|_{r=r_{0}}+\left.\varepsilon \frac{\partial^{2} U_{n}^{(\mathrm{i})}}{\partial r^{2}}\right|_{r=r_{0}} r_{0} \cos 2 \theta+\left.2 \varepsilon \frac{\sin 2 \theta}{r_{0}} \frac{\partial U_{n}^{(\mathrm{i})}}{\partial \theta}\right|_{r=r_{0}} \\
=\left.\frac{\partial U_{n}^{(\mathrm{o})}}{\partial r}\right|_{r=r_{0}}+\left.\varepsilon \frac{\partial^{2} U_{n}^{(\mathrm{o})}}{\partial r^{2}}\right|_{r=r_{0}} r_{0} \cos 2 \theta+\left.2 \varepsilon \frac{\sin 2 \theta}{r_{0}} \frac{\partial U_{n}^{(\mathrm{o})}}{\partial \theta}\right|_{r=r_{0}} .
\end{gathered}
$$

Here, ${ }^{(i)}$ and ${ }^{(0)}$ denote the inside and outside of $\Omega_{\mathrm{c}}$, respectively. Note that (A.5)-(A.7) are derived from (36), in particular (A.6) and (A.7) are from the Taylor expansion of the following equations, respectively:

$$
\begin{aligned}
U^{(\mathrm{i})}\left(r_{0}(1+\varepsilon \cos 2 \theta), \theta\right) & =U^{(\mathrm{o})}\left(r_{0}(1+\varepsilon \cos 2 \theta), \theta\right), \\
\left(\left.\nabla U_{n}^{(\mathrm{i})}\right|_{r=r_{0}(1+\varepsilon \cos 2 \theta)}\right) \cdot \boldsymbol{n}(\theta) & =\left(\left.\nabla U_{n}^{(\mathrm{o})}\right|_{r=r_{0}(1+\varepsilon \cos 2 \theta)}\right) \cdot \boldsymbol{n}(\theta) .
\end{aligned}
$$


We consider the general solutions of (A.2) and (A.3). Since (A.2) and (A.3) are linear equations, their general solutions $U_{n}^{(\mathrm{g})}(r, \theta ; \varepsilon)$ can be written as

$$
U_{n}^{(\mathrm{g})}(r, \theta ; \varepsilon)=U_{n}^{(\mathrm{p})}(r, \theta ; \varepsilon)+U^{(\mathrm{c})}(r, \theta ; \varepsilon),
$$

where $U_{n}^{(\mathrm{p})}(r, \theta ; \varepsilon)$ is a particular solution of $(\mathrm{A} .2)$ or $(\mathrm{A} .3)$ and $U^{(\mathrm{c})}(r, \theta ; \varepsilon)$ is the general solution of the complementary equation of (A.2) or (A.3):

$$
\frac{\partial^{2} U}{\partial r^{2}}+\frac{1}{r} \frac{\partial U}{\partial r}+\frac{1}{r^{2}} \frac{\partial^{2} U}{\partial \theta^{2}}-U=0
$$

(A.8) can be solved by separation of variables and the solution can be written as follows:

$$
U^{(\mathrm{c})}(r, \theta ; \varepsilon)=\sum_{k=0}^{\infty}\left[\left(A_{k} \mathcal{I}_{k}(r)+B_{k} \mathcal{K}_{k}(r)\right) \cos k \theta+\left(C_{k} \mathcal{I}_{k}(r)+D_{k} \mathcal{K}_{k}(r)\right) \sin k \theta\right],
$$

where $A_{k}, B_{k}, C_{k}$ and $D_{k}$ are arbitrary constants. We can also find $U_{n}^{(\mathrm{p})}(r, \theta ; \varepsilon)$ : e.g.,

$$
\begin{gathered}
U_{0}^{(\mathrm{p})(\mathrm{i})}(r, \theta ; \varepsilon)=0, \\
U_{0}^{(\mathrm{p})(\mathrm{o})}(r, \theta ; \varepsilon)=1, \\
U_{1}^{(\mathrm{p})(\mathrm{i})}(r, \theta ; \varepsilon)=1-r_{0} \mathcal{K}_{1}\left(r_{0}\right) \mathcal{I}_{0}(r), \\
U_{1}^{(\mathrm{p})(\mathrm{o})}(r, \theta ; \varepsilon)=r_{0} \mathcal{I}_{1}\left(r_{0}\right) \mathcal{K}_{0}(r) .
\end{gathered}
$$

Next, we consider the solutions of (A.2) and (A.3) under the conditions (A.4)-(A.7). Imposing (A.4) and (A.5) on $U_{n}^{(\mathrm{g})}(r, \theta ; \varepsilon)$, we can describe $U_{n}(r, \theta ; \varepsilon)$ as follows:

$$
\begin{aligned}
U_{n}^{(\mathrm{i})}(r, \theta ; \varepsilon) & =U_{n}^{(\mathrm{p})(\mathrm{i})}(r, \theta ; \varepsilon)+\sum_{k=0}^{\infty}\left(A_{n, k} \mathcal{I}_{k}(r) \cos k \theta+C_{n, k} \mathcal{I}_{k}(r) \sin k \theta\right), \\
U_{n}^{(\mathrm{o})}(r, \theta ; \varepsilon) & =U_{n}^{(\mathrm{p})(\mathrm{o})}(r, \theta ; \varepsilon)+\sum_{k=0}^{\infty}\left(B_{n, k} \mathcal{K}_{k}(r) \cos k \theta+D_{n, k} \mathcal{K}_{k}(r) \sin k \theta\right),
\end{aligned}
$$

where $A_{n, k}, B_{n, k}, C_{n, k}$ and $D_{n, k}$ are the constants to be determined by (A.6) and (A.7).

Finally, to determine the constants $A_{n, k}, B_{n, k}, C_{n, k}$ and $D_{n, k}$, we expand each with respect to small $\varepsilon$ :

$$
\begin{aligned}
& A_{n, k}(\varepsilon)=a_{n, k}+\varepsilon \tilde{a}_{n, k}+O\left(\varepsilon^{2}\right), \\
& B_{n, k}(\varepsilon)=b_{n, k}+\varepsilon \tilde{b}_{n, k}+O\left(\varepsilon^{2}\right), \\
& C_{n, k}(\varepsilon)=c_{n, k}+\varepsilon \tilde{c}_{n, k}+O\left(\varepsilon^{2}\right), \\
& D_{n, k}(\varepsilon)=d_{n, k}+\varepsilon \tilde{d}_{n, k}+O\left(\varepsilon^{2}\right) .
\end{aligned}
$$


Setting $\varepsilon=0$ in (A.6) and (A.7), we obtain $a_{n, k}, b_{n, k}, c_{n, k}$ and $d_{n, k}$ : e.g.,

$$
\begin{gathered}
\left\{\begin{array}{l}
a_{0, k}=b_{0, k}=0 \quad(k \neq 0, k=1,2, \cdots), \\
c_{0, k}=d_{0, k}=0 \quad(k=0,1,2, \cdots), \\
a_{0,0}=-r_{0} \mathcal{K}_{1}\left(r_{0}\right), \quad b_{0,0}=r_{0} \mathcal{I}_{1}\left(r_{0}\right),
\end{array}\right. \\
\left\{\begin{array}{l}
a_{1, k}=b_{1, k}=0 \quad(k \neq 1, k=0,2,3,4, \cdots), \\
c_{1, k}=d_{1, k}=0 \quad(k=0,1,2, \cdots), \\
a_{1,1}=-\frac{r_{0}^{2}}{2} \mathcal{K}_{2}\left(r_{0}\right), \quad b_{1,1}=\frac{r_{0}^{2}}{2} \mathcal{I}_{2}\left(r_{0}\right) .
\end{array}\right.
\end{gathered}
$$

When considering (A.6) and (A.7) within the first order of $\varepsilon$, we obtain $\tilde{a}_{n, k}, \tilde{b}_{n, k}, \tilde{c}_{n, k}$ and $\tilde{d}_{n, k}$ : e.g.,

$$
\begin{gathered}
\left\{\begin{array}{l}
\tilde{a}_{0, k}=\tilde{b}_{0, k}=0 \quad(k \neq 2, k=0,1,3,4,5, \cdots), \\
\tilde{c}_{0, k}=\tilde{d}_{0, k}=0 \quad(k=0,1,2, \cdots), \\
\tilde{a}_{0,2}=r_{0}^{2} \mathcal{K}_{2}\left(r_{0}\right), \quad \tilde{b}_{0,2}=r_{0}^{2} \mathcal{I}_{2}\left(r_{0}\right),
\end{array}\right. \\
\left\{\begin{array}{l}
\tilde{a}_{1, k}=\tilde{b}_{1, k}=0 \quad(k \neq 1,3, k=0,2,4,5,6, \cdots), \\
\tilde{c}_{1, k}=\tilde{d}_{1, k}=0 \quad(k=0,1,2, \cdots), \\
\tilde{a}_{1,1}=\frac{r_{0}^{3}}{4} \mathcal{K}_{1}\left(r_{0}\right), \quad \tilde{b}_{1,1}=\frac{r_{0}^{3}}{4} \mathcal{I}_{1}\left(r_{0}\right), \quad \tilde{a}_{1,3}=\frac{r_{0}^{3}}{4} \mathcal{K}_{3}\left(r_{0}\right), \quad \tilde{b}_{1,3}=\frac{r_{0}^{3}}{4} \mathcal{I}_{3}\left(r_{0}\right) .
\end{array}\right.
\end{gathered}
$$

Substituting the above results into (A.9) and (A.10), we obtain $U_{n}(r, \theta ; \varepsilon)$, which we rewrite as $U_{n}(r, \theta)+\varepsilon \tilde{U}_{n}(r, \theta)+O\left(\varepsilon^{2}\right)$. The results can be further simplified by using the formulae of modified Bessel functions [33], and we finally obtain

$$
\begin{gathered}
U_{0}^{(\mathrm{i})}(r, \theta)=1-r_{0} \mathcal{K}_{1}\left(r_{0}\right) \mathcal{I}_{0}(r), \\
U_{0}^{(\mathrm{o})}(r, \theta)=r_{0} \mathcal{I}_{1}\left(r_{0}\right) \mathcal{K}_{0}(r), \\
\tilde{U}_{0}^{(\mathrm{i})}(r, \theta)=r_{0}^{2} \mathcal{K}_{2}\left(r_{0}\right) \mathcal{I}_{2}(r) \cos 2 \theta, \\
\tilde{U}_{0}^{(\mathrm{o})}(r, \theta)=r_{0}^{2} \mathcal{I}_{2}\left(r_{0}\right) \mathcal{K}_{2}(r) \cos 2 \theta, \\
U_{1}^{(\mathrm{i})}(r, \theta)=\frac{r_{0}^{2} r}{4}\left(-\mathcal{K}_{0}\left(r_{0}\right) \mathcal{I}_{0}(r)+\mathcal{K}_{2}\left(r_{0}\right) \mathcal{I}_{2}(r)\right) \cos \theta, \\
U_{1}^{(\mathrm{o})}(r, \theta)=\frac{r_{0}^{2} r}{4}\left(-\mathcal{I}_{0}\left(r_{0}\right) \mathcal{K}_{0}(r)+\mathcal{I}_{2}\left(r_{0}\right) \mathcal{K}_{2}(r)\right) \cos \theta,
\end{gathered}
$$




$$
\begin{aligned}
\tilde{U}_{1}^{(\mathrm{i})}(r, \theta) & =\frac{r_{0}^{2}}{4}\left(r_{0} \mathcal{K}_{1}\left(r_{0}\right) \mathcal{I}_{1}(r)-r \mathcal{K}_{2}\left(r_{0}\right) \mathcal{I}_{2}(r)\right) \cos \theta \\
& +\frac{r_{0}^{2}}{4}\left(-r \mathcal{K}_{2}\left(r_{0}\right) \mathcal{I}_{2}(r)+r_{0} \mathcal{K}_{3}\left(r_{0}\right) \mathcal{I}_{3}(r)\right) \cos 3 \theta \\
\tilde{U}_{1}^{(\mathrm{o})}(r, \theta) & =\frac{r_{0}^{2}}{4}\left(r_{0} \mathcal{I}_{1}\left(r_{0}\right) \mathcal{K}_{1}(r)-r \mathcal{I}_{2}\left(r_{0}\right) \mathcal{K}_{2}(r)\right) \cos \theta \\
& +\frac{r_{0}^{2}}{4}\left(-r \mathcal{I}_{2}\left(r_{0}\right) \mathcal{K}_{2}(r)+r_{0} \mathcal{I}_{3}\left(r_{0}\right) \mathcal{K}_{3}(r)\right) \cos 3 \theta
\end{aligned}
$$

$$
\begin{aligned}
U_{2}^{(\mathrm{i})}(r, \theta) & =\frac{r_{0}^{2}}{32}\left(r^{2} \mathcal{K}_{0}\left(r_{0}\right) \mathcal{I}_{0}(r)-r^{2} \mathcal{K}_{2}\left(r_{0}\right) \mathcal{I}_{2}(r)-2 r_{0} \mathcal{K}_{1}\left(r_{0}\right) \mathcal{I}_{0}(r)+2 r \mathcal{K}_{0}\left(r_{0}\right) \mathcal{I}_{1}(r)\right) \\
& +\frac{r_{0}^{2}}{64}\left(2 r^{2} \mathcal{K}_{0}\left(r_{0}\right) \mathcal{I}_{0}(r)-r_{0} r \mathcal{K}_{1}\left(r_{0}\right) \mathcal{I}_{1}(r)-2 r^{2} \mathcal{K}_{2}\left(r_{0}\right) \mathcal{I}_{2}(r)+r_{0} r \mathcal{K}_{3}\left(r_{0}\right) \mathcal{I}_{3}(r)\right) \cos 2 \theta \\
U_{2}^{(\mathrm{o})}(r, \theta) & =\frac{r_{0}^{2}}{32}\left(r^{2} \mathcal{I}_{0}\left(r_{0}\right) \mathcal{K}_{0}(r)-r^{2} \mathcal{I}_{2}\left(r_{0}\right) \mathcal{K}_{2}(r)+2 r_{0} \mathcal{I}_{1}\left(r_{0}\right) \mathcal{K}_{0}(r)-2 r \mathcal{I}_{0}\left(r_{0}\right) \mathcal{K}_{1}(r)\right) \\
& +\frac{r_{0}^{2}}{64}\left(2 r^{2} \mathcal{I}_{0}\left(r_{0}\right) \mathcal{K}_{0}(r)-r_{0} r \mathcal{I}_{1}\left(r_{0}\right) \mathcal{K}_{1}(r)-2 r^{2} \mathcal{I}_{2}\left(r_{0}\right) \mathcal{K}_{2}(r)+r_{0} r \mathcal{I}_{3}\left(r_{0}\right) \mathcal{K}_{3}(r)\right) \cos 2 \theta
\end{aligned}
$$

$$
\begin{aligned}
\tilde{U}_{2}^{(\mathrm{i})}(r, \theta) & =\frac{r_{0}^{2}}{32}\left(r_{0}^{2} \mathcal{K}_{0}\left(r_{0}\right) \mathcal{I}_{0}(r)-2 r_{0} r \mathcal{K}_{1}\left(r_{0}\right) \mathcal{I}_{1}(r)+r^{2} \mathcal{K}_{2}\left(r_{0}\right) \mathcal{I}_{2}(r)\right) \\
& +\frac{r_{0}^{2}}{32}\left\{-3 r_{0} r \mathcal{K}_{1}\left(r_{0}\right) \mathcal{I}_{1}(r)+2\left(r_{0}^{2}+r^{2}\right) \mathcal{K}_{2}\left(r_{0}\right) \mathcal{I}_{2}(r)-r_{0} r \mathcal{K}_{3}\left(r_{0}\right) \mathcal{I}_{3}(r)\right\} \cos 2 \theta \\
& +\frac{r_{0}^{2}}{32}\left(r^{2} \mathcal{K}_{2}\left(r_{0}\right) \mathcal{I}_{2}(r)-2 r_{0} r \mathcal{K}_{3}\left(r_{0}\right) \mathcal{I}_{3}(r)+r_{0}^{2} \mathcal{K}_{4}\left(r_{0}\right) \mathcal{I}_{4}(r)\right) \cos 4 \theta \\
\tilde{U}_{2}^{(o)}(r, \theta)= & \frac{r_{0}^{2}}{32}\left(r_{0}^{2} \mathcal{I}_{0}\left(r_{0}\right) \mathcal{K}_{0}(r)-2 r_{0} r \mathcal{I}_{1}\left(r_{0}\right) \mathcal{K}_{1}(r)+r^{2} \mathcal{I}_{2}\left(r_{0}\right) \mathcal{K}_{2}(r)\right) \\
& +\frac{r_{0}^{2}}{32}\left\{-3 r_{0} r \mathcal{I}_{1}\left(r_{0}\right) \mathcal{K}_{1}(r)+2\left(r_{0}^{2}+r^{2}\right) \mathcal{I}_{2}\left(r_{0}\right) \mathcal{K}_{2}(r)-r_{0} r \mathcal{I}_{3}\left(r_{0}\right) \mathcal{K}_{3}(r)\right\} \cos 2 \theta \\
& +\frac{r_{0}^{2}}{32}\left(r^{2} \mathcal{I}_{2}\left(r_{0}\right) \mathcal{K}_{2}(r)-2 r_{0} r \mathcal{I}_{3}\left(r_{0}\right) \mathcal{K}_{3}(r)+r_{0}^{2} \mathcal{I}_{4}\left(r_{0}\right) \mathcal{K}_{4}(r)\right) \cos 4 \theta
\end{aligned}
$$




$$
\begin{aligned}
& U_{3}^{(\mathrm{i})}(r, \theta)=\frac{r_{0}^{2} r}{128}\left\{-\left(r_{0}^{2}+r^{2}\right) \mathcal{K}_{0}\left(r_{0}\right) \mathcal{I}_{0}(r)+\left(r_{0}^{2}+r^{2}\right) \mathcal{K}_{2}\left(r_{0}\right) \mathcal{I}_{2}(r)\right. \\
& \left.+4 r_{0} \mathcal{K}_{1}\left(r_{0}\right) \mathcal{I}_{0}(r)-4 r \mathcal{K}_{0}\left(r_{0}\right) \mathcal{I}_{1}(r)\right\} \cos \theta \\
& +\frac{r_{0}^{2} r}{1152}\left\{-3 r^{2} \mathcal{K}_{0}\left(r_{0}\right) \mathcal{I}_{0}(r)+3 r_{0} r \mathcal{K}_{1}\left(r_{0}\right) \mathcal{I}_{1}(r)+\left(3 r^{2}-r_{0}^{2}\right) \mathcal{K}_{2}\left(r_{0}\right) \mathcal{I}_{2}(r)\right. \\
& \left.-3 r_{0} r \mathcal{K}_{3}\left(r_{0}\right) \mathcal{I}_{3}(r)+r_{0}^{2} \mathcal{K}_{4}\left(r_{0}\right) \mathcal{I}_{4}(r)\right\} \cos 3 \theta \\
& U_{3}^{(\mathrm{o})}(r, \theta)=\frac{r_{0}^{2} r}{128}\left\{-\left(r_{0}^{2}+r^{2}\right) \mathcal{I}_{0}\left(r_{0}\right) \mathcal{K}_{0}(r)+\left(r_{0}^{2}+r^{2}\right) \mathcal{I}_{2}\left(r_{0}\right) \mathcal{K}_{2}(r)\right. \\
& \left.-4 r_{0} \mathcal{I}_{1}\left(r_{0}\right) \mathcal{K}_{0}(r)+4 r \mathcal{I}_{0}\left(r_{0}\right) \mathcal{K}_{1}(r)\right\} \cos \theta \\
& +\frac{r_{0}^{2} r}{1152}\left\{-3 r^{2} \mathcal{I}_{0}\left(r_{0}\right) \mathcal{K}_{0}(r)+3 r_{0} r \mathcal{I}_{1}\left(r_{0}\right) \mathcal{K}_{1}(r)+\left(3 r^{2}-r_{0}^{2}\right) \mathcal{I}_{2}\left(r_{0}\right) \mathcal{K}_{2}(r)\right. \\
& \left.-3 r_{0} r \mathcal{I}_{3}\left(r_{0}\right) \mathcal{K}_{3}(r)+r_{0}^{2} \mathcal{I}_{4}\left(r_{0}\right) \mathcal{K}_{4}(r)\right\} \cos 3 \theta \\
& \tilde{U}_{3}^{(\mathrm{i})}(r, \theta)=\frac{r_{0}^{2}}{96}\left\{-3 r_{0}^{2} r \mathcal{K}_{0}\left(r_{0}\right) \mathcal{I}_{0}(r)+r_{0}\left(3 r^{2}+r_{0}^{2}\right) \mathcal{K}_{1}\left(r_{0}\right) \mathcal{I}_{1}(r)-r^{3} \mathcal{K}_{2}\left(r_{0}\right) \mathcal{I}_{2}(r)\right\} \cos \theta \\
& +\frac{r_{0}^{2}}{384}\left\{6 r_{0} r^{2} \mathcal{K}_{1}\left(r_{0}\right) \mathcal{I}_{1}(r)-r\left(8 r_{0}^{2}+3 r^{2}\right) \mathcal{K}_{2}\left(r_{0}\right) \mathcal{I}_{2}(r)+3 r_{0}\left(r^{2}+r_{0}^{2}\right) \mathcal{K}_{3}\left(r_{0}\right) \mathcal{I}_{3}(r)\right. \\
& \left.-r_{0}^{2} r \mathcal{K}_{4}\left(r_{0}\right) \mathcal{I}_{4}(r)\right\} \cos 3 \theta \\
& +\frac{r_{0}^{2}}{384}\left(-r^{3} \mathcal{K}_{2}\left(r_{0}\right) \mathcal{I}_{2}(r)+3 r_{0} r^{2} \mathcal{K}_{3}\left(r_{0}\right) \mathcal{I}_{3}(r)-3 r_{0}^{2} r \mathcal{K}_{4}\left(r_{0}\right) \mathcal{I}_{4}(r)+r_{0}^{3} \mathcal{K}_{5}\left(r_{0}\right) \mathcal{I}_{5}(r)\right) \cos 5 \theta \\
& \tilde{U}_{3}^{(\mathrm{o})}(r, \theta)=\frac{r_{0}^{2}}{96}\left\{-3 r_{0}^{2} r \mathcal{I}_{0}\left(r_{0}\right) \mathcal{K}_{0}(r)+r_{0}\left(3 r^{2}+r_{0}^{2}\right) \mathcal{I}_{1}\left(r_{0}\right) \mathcal{K}_{1}(r)-r^{3} \mathcal{I}_{2}\left(r_{0}\right) \mathcal{K}_{2}(r)\right\} \cos \theta \\
& +\frac{r_{0}^{2}}{384}\left\{6 r_{0} r^{2} \mathcal{I}_{1}\left(r_{0}\right) \mathcal{K}_{1}(r)-r\left(8 r_{0}^{2}+3 r^{2}\right) \mathcal{I}_{2}\left(r_{0}\right) \mathcal{K}_{2}(r)+3 r_{0}\left(r^{2}+r_{0}^{2}\right) \mathcal{I}_{3}\left(r_{0}\right) \mathcal{K}_{3}(r)\right. \\
& \left.-r_{0}^{2} r \mathcal{I}_{4}\left(r_{0}\right) \mathcal{K}_{4}(r)\right\} \cos 3 \theta \\
& +\frac{r_{0}^{2}}{384}\left(-r^{3} \mathcal{I}_{2}\left(r_{0}\right) \mathcal{K}_{2}(r)+3 r_{0} r^{2} \mathcal{I}_{3}\left(r_{0}\right) \mathcal{K}_{3}(r)-3 r_{0}^{2} r \mathcal{I}_{4}\left(r_{0}\right) \mathcal{K}_{4}(r)+r_{0}^{3} \mathcal{I}_{5}\left(r_{0}\right) \mathcal{K}_{5}(r)\right) \cos 5 \theta
\end{aligned}
$$

\section{Appendix B. Perturbation method for the rotating solution}

To obtain $V_{n}$ and $\tilde{V}_{n}(n=0,1,2, \cdots)$ in (59), we first consider the following expansion of $V(r, \theta ; \varepsilon, \omega)$ :

$$
V(r, \theta ; \varepsilon, \omega)=\sum_{n=0}^{\infty} V_{n}(r, \theta ; \varepsilon) \omega^{n},
$$

where $V_{0}(r, \theta ; \varepsilon)=U_{0}(r, \theta ; \varepsilon)$ as derived in Appendix $\mathrm{A}$ and $V_{n}(r, \theta ; \varepsilon)(n=1,2, \cdots)$ are the functions satisfying the following equation:

$$
-\frac{\partial V_{n-1}}{\partial \theta}=\frac{\partial^{2} V_{n}}{\partial r^{2}}+\frac{1}{r} \frac{\partial V_{n}}{\partial r}+\frac{1}{r^{2}} \frac{\partial^{2} V_{n}}{\partial \theta^{2}}-V_{n}
$$


as well as the following conditions:

$$
\begin{gathered}
\lim _{r \rightarrow \infty} V_{n}(r, \theta)=0 \\
\lim _{r \rightarrow+0} V_{n}(r, \theta)<\infty, \quad \lim _{r \rightarrow+0} \frac{\partial V_{n}}{\partial r}(r, \theta)=0 \\
V_{n}^{(\mathrm{i})}\left(r_{0}, \theta\right)+\left.\varepsilon \frac{\partial V_{n}^{(\mathrm{i})}}{\partial r}\right|_{r=r_{0}} r_{0} \cos 2 \theta=V_{n}^{(\mathrm{o})}\left(r_{0}, \theta\right)+\left.\varepsilon \frac{\partial V_{n}^{(\mathrm{o})}}{\partial r}\right|_{r=r_{0}} r_{0} \cos 2 \theta, \\
\left.\frac{\partial V_{n}^{(\mathrm{i})}}{\partial r}\right|_{r=r_{0}}+\left.\varepsilon \frac{\partial^{2} V_{n}^{(\mathrm{i})}}{\partial r^{2}}\right|_{r=r_{0}} r_{0} \cos 2 \theta+\left.2 \varepsilon \frac{\sin 2 \theta}{r_{0}} \frac{\partial V_{n}^{(\mathrm{i})}}{\partial \theta}\right|_{r=r_{0}} \\
=\left.\frac{\partial V_{n}^{(\mathrm{o})}}{\partial r}\right|_{r=r_{0}}+\left.\varepsilon \frac{\partial^{2} V_{n}^{(\mathrm{o})}}{\partial r^{2}}\right|_{r=r_{0}} r_{0} \cos 2 \theta+\left.2 \varepsilon \frac{\sin 2 \theta}{r_{0}} \frac{\partial V_{n}^{(\mathrm{o})}}{\partial \theta}\right|_{r=r_{0}} .
\end{gathered}
$$

Here, ${ }^{(i)}$ and ${ }^{(o)}$ denote the inside and outside of $\Omega_{\mathrm{c}}$, respectively. Note that (B.4)-(B.6) are derived from (52), in particular (B.5) and (B.6) are from the Taylor expansion of the following equations, respectively:

$$
\begin{aligned}
V^{(\mathrm{i})}\left(r_{0}(1+\varepsilon \cos 2 \theta), \theta\right) & =V^{(\mathrm{o})}\left(r_{0}(1+\varepsilon \cos 2 \theta), \theta\right) \\
\left(\left.\nabla V_{n}^{(\mathrm{i})}\right|_{r=r_{0}(1+\varepsilon \cos 2 \theta)}\right) \cdot \boldsymbol{n}(\theta) & =\left(\left.\nabla V_{n}^{(\mathrm{o})}\right|_{r=r_{0}(1+\varepsilon \cos 2 \theta)}\right) \cdot \boldsymbol{n}(\theta) .
\end{aligned}
$$

We consider the general solution of (B.2). Since (B.2) is linear equation, its general solution $V_{n}^{(\mathrm{g})}(r, \theta ; \varepsilon)$ can be written as

$$
V_{n}^{(\mathrm{g})}(r, \theta ; \varepsilon)=V_{n}^{(\mathrm{p})}(r, \theta ; \varepsilon)+V^{(\mathrm{c})}(r, \theta ; \varepsilon),
$$

where $V_{n}^{(\mathrm{p})}(r, \theta ; \varepsilon)$ is a particular solution of $(\mathrm{B} .2)$ and $V^{(\mathrm{c})}(r, \theta ; \varepsilon)$ is the general solution of the complementary equation of (B.2):

$$
\frac{\partial^{2} V}{\partial r^{2}}+\frac{1}{r} \frac{\partial V}{\partial r}+\frac{1}{r^{2}} \frac{\partial^{2} V}{\partial \theta^{2}}-V=0
$$

(B.7) can be solved by separation of variables and the solution can be written as follows:

$$
V^{(\mathrm{c})}(r, \theta ; \varepsilon)=\sum_{k=0}^{\infty}\left[\left(A_{k} \mathcal{I}_{k}(r)+B_{k} \mathcal{K}_{k}(r)\right) \cos k \theta+\left(C_{k} \mathcal{I}_{k}(r)+D_{k} \mathcal{K}_{k}(r)\right) \sin k \theta\right],
$$

where $A_{k}, B_{k}, C_{k}$ and $D_{k}$ are arbitrary constants. We can also find $V_{n}^{(\mathrm{p})}(r, \theta ; \varepsilon)$ : e.g.,

$$
\begin{gathered}
V_{0}^{(\mathrm{p})(\mathrm{i})}(r, \theta ; \varepsilon)=0, \\
V_{0}^{(\mathrm{p})(\mathrm{o})}(r, \theta ; \varepsilon)=1,
\end{gathered}
$$




$$
\begin{aligned}
V_{1}^{(\mathrm{p})(\mathrm{i})}(r, \theta ; \varepsilon) & =1-r_{0} \mathcal{K}_{1}\left(r_{0}\right) \mathcal{I}_{0}(r), \\
V_{1}^{(\mathrm{p})(\mathrm{o})}(r, \theta ; \varepsilon) & =r_{0} \mathcal{I}_{1}\left(r_{0}\right) \mathcal{K}_{0}(r) .
\end{aligned}
$$

Next, we consider the solutions of (B.2) under the conditions (B.3)-(B.6). Imposing (B.3) and (B.4) on $V_{n}^{(\mathrm{g})}(r, \theta ; \varepsilon)$, we can describe $V_{n}(r, \theta ; \varepsilon)$ as follows:

$$
\begin{aligned}
& V_{n}^{(\mathrm{i})}(r, \theta ; \varepsilon)=V_{n}^{(\mathrm{p})(\mathrm{i})}(r, \theta ; \varepsilon)+\sum_{k=0}^{\infty}\left(A_{n, k} \mathcal{I}_{k}(r) \cos k \theta+C_{n, k} \mathcal{I}_{k}(r) \sin k \theta\right), \\
& V_{n}^{(\mathrm{o})}(r, \theta ; \varepsilon)=V_{n}^{(\mathrm{p})(\mathrm{o})}(r, \theta ; \varepsilon)+\sum_{k=0}^{\infty}\left(B_{n, k} \mathcal{K}_{k}(r) \cos k \theta+D_{n, k} \mathcal{K}_{k}(r) \sin k \theta\right),
\end{aligned}
$$

where $A_{n, k}, B_{n, k}, C_{n, k}$ and $D_{n, k}$ are the constants to be determined by (B.5) and (B.6).

Finally, to determine the constants $A_{n, k}, B_{n, k}, C_{n, k}$ and $D_{n, k}$, we expand each with respect to small $\varepsilon$ :

$$
\begin{aligned}
& A_{n, k}(\varepsilon)=a_{n, k}+\varepsilon \tilde{a}_{n, k}+O\left(\varepsilon^{2}\right), \\
& B_{n, k}(\varepsilon)=b_{n, k}+\varepsilon \tilde{b}_{n, k}+O\left(\varepsilon^{2}\right), \\
& C_{n, k}(\varepsilon)=c_{n, k}+\varepsilon \tilde{c}_{n, k}+O\left(\varepsilon^{2}\right), \\
& D_{n, k}(\varepsilon)=d_{n, k}+\varepsilon \tilde{d}_{n, k}+O\left(\varepsilon^{2}\right) .
\end{aligned}
$$

When considering (B.5) and (B.6) within the first order of $\varepsilon$, we obtain $a_{n, k}, b_{n, k}, c_{n, k}, d_{n, k}, \tilde{a}_{n, k}$, $\tilde{b}_{n, k}, \tilde{c}_{n, k}$ and $\tilde{d}_{n, k}$ : e.g.

$$
\begin{aligned}
& \left\{\begin{array}{l}
a_{1, k}=b_{1, k}=c_{1, k}=d_{1, k}=0 \quad(k=0,1,2, \cdots), \\
\tilde{a}_{1, k}=\tilde{b}_{1, k}=0 \quad(k=0,1,2, \cdots), \\
\tilde{c}_{1, k}=\tilde{d}_{1, k}=0 \quad(k \neq 2, k=0,1,3,4,5, \cdots), \\
\tilde{c}_{1,2}=-r_{0}^{3} \mathcal{K}_{3}\left(r_{0}\right), \quad \tilde{d}_{1,2}=r_{0}^{3} \mathcal{I}_{3}\left(r_{0}\right),
\end{array}\right. \\
& \left\{\begin{array}{l}
a_{2, k}=b_{2, k}=c_{2, k}=d_{2, k}=0 \quad(k=0,1,2, \cdots), \\
\tilde{a}_{2, k}=\tilde{b}_{2, k}=0 \quad(k \neq 2, k=0,1,3,4,5, \cdots), \\
\tilde{c}_{2, k}=\tilde{d}_{2, k}=0 \quad(k=0,1,2, \cdots), \\
\tilde{a}_{2,2}=-\frac{r_{0}^{4}}{2} \mathcal{K}_{4}\left(r_{0}\right), \quad \tilde{b}_{2,2}=-\frac{r_{0}^{4}}{2} \mathcal{I}_{4}\left(r_{0}\right) .
\end{array}\right.
\end{aligned}
$$

Substituting the above results into (B.8) and (B.9), we obtain $V_{n}(r, \theta ; \varepsilon)$, which we rewrite as $V_{n}(r, \theta)+\varepsilon \tilde{V}_{n}(r, \theta)+O\left(\varepsilon^{2}\right)$. The results can be further simplified by using the formulae of modified 
Bessel functions [33], and we finally obtain

$$
\begin{aligned}
& V_{0}^{(\mathrm{i})}(r, \theta)=1-r_{0} \mathcal{K}_{1}\left(r_{0}\right) \mathcal{I}_{0}(r), \\
& V_{0}^{(\mathrm{o})}(r, \theta)=r_{0} \mathcal{I}_{1}\left(r_{0}\right) \mathcal{K}_{0}(r), \\
& \tilde{V}_{0}^{(\mathrm{i})}(r, \theta)=r_{0}^{2} \mathcal{K}_{2}\left(r_{0}\right) \mathcal{I}_{2}(r) \cos 2 \theta, \\
& \tilde{V}_{0}^{(o)}(r, \theta)=r_{0}^{2} \mathcal{I}_{2}\left(r_{0}\right) \mathcal{K}_{2}(r) \cos 2 \theta \text {, } \\
& V_{1}^{(\mathrm{i})}(r, \theta)=0, \\
& V_{1}^{(\mathrm{o})}(r, \theta)=0 \text {, } \\
& \tilde{V}_{1}^{(\mathrm{i})}(r, \theta)=\frac{r_{0}^{3} r}{4}\left(-\mathcal{K}_{1}\left(r_{0}\right) \mathcal{I}_{1}(r)+\mathcal{K}_{3}\left(r_{0}\right) \mathcal{I}_{3}(r)\right) \sin 2 \theta, \\
& \tilde{V}_{1}^{(\mathrm{o})}(r, \theta)=\frac{r_{0}^{3} r}{4}\left(-\mathcal{I}_{1}\left(r_{0}\right) \mathcal{K}_{1}(r)+\mathcal{I}_{3}\left(r_{0}\right) \mathcal{K}_{3}(r)\right) \sin 2 \theta, \\
& V_{2}^{(\mathrm{i})}(r, \theta)=0, \\
& V_{2}^{(\mathrm{o})}(r, \theta)=0 \text {, } \\
& \tilde{V}_{2}^{(\mathrm{i})}(r, \theta)=\frac{r_{0}^{4} r^{2}}{48}\left(-3 \mathcal{K}_{0}\left(r_{0}\right) \mathcal{I}_{0}(r)+4 \mathcal{K}_{2}\left(r_{0}\right) \mathcal{I}_{2}(r)-\mathcal{K}_{4}\left(r_{0}\right) \mathcal{I}_{4}(r)\right) \cos 2 \theta, \\
& \tilde{V}_{2}^{(o)}(r, \theta)=\frac{r_{0}^{4} r^{2}}{48}\left(-3 \mathcal{I}_{0}\left(r_{0}\right) \mathcal{K}_{0}(r)+4 \mathcal{I}_{2}\left(r_{0}\right) \mathcal{K}_{2}(r)-\mathcal{I}_{4}\left(r_{0}\right) \mathcal{K}_{4}(r)\right) \cos 2 \theta, \\
& V_{3}^{(\mathrm{i})}(r, \theta)=0, \\
& V_{3}^{(\mathrm{o})}(r, \theta)=0 \text {, } \\
& \tilde{V}_{3}^{(\mathrm{i})}(r, \theta)=\frac{r_{0}^{3} r}{72}\left\{3\left(r_{0}^{2}+r^{2}\right) \mathcal{K}_{1}\left(r_{0}\right) \mathcal{I}_{1}(r)-4 r_{0} r \mathcal{K}_{2}\left(r_{0}\right) \mathcal{I}_{2}(r)-3\left(r_{0}^{2}+r^{2}\right) \mathcal{K}_{3}\left(r_{0}\right) \mathcal{I}_{3}(r)\right. \\
& \left.+4 r_{0} r \mathcal{K}_{4}\left(r_{0}\right) \mathcal{I}_{4}(r)\right\} \sin 2 \theta, \\
& \tilde{V}_{3}^{(\mathrm{o})}(r, \theta)=\frac{r_{0}^{3} r}{72}\left\{3\left(r_{0}^{2}+r^{2}\right) \mathcal{I}_{1}\left(r_{0}\right) \mathcal{K}_{1}(r)-4 r_{0} r \mathcal{I}_{2}\left(r_{0}\right) \mathcal{K}_{2}(r)-3\left(r_{0}^{2}+r^{2}\right) \mathcal{I}_{3}\left(r_{0}\right) \mathcal{K}_{3}(r)\right. \\
& \left.+4 r_{0} r \mathcal{I}_{4}\left(r_{0}\right) \mathcal{K}_{4}(r)\right\} \sin 2 \theta .
\end{aligned}
$$




\section{References}

[1] R. Mallik and S. P. Gross, Molecular Motors: Strategies to Get Along, Curr. Biol. 14 (2004) 971-982.

[2] D. Kaiser, Bacterial Swarming: A Re-examination of Cell-Movement Patterns, Curr. Biol. 17 (2007) 561-570.

[3] W. F. Paxton, A. Sen and T. E. Mallouk, Motility of Catalytic Nanoparticles through SelfGenerated Forces, Chem. Eur. J 11 (2005) 6462-6470.

[4] S. Nakata, Y. Iguchi, S. Ose, M. Kuboyama, T. Ishii and K. Yoshikawa, Self-Rotation of a Camphor Scraping on Water: New Insight into the Old Problem, Langmuir 13 (1997) 44544458.

[5] S. Nakata, Y. Iguchi, S. Ose and T. Ishii, pH-Sensitive Self-Motion of a Solid Scraping on an Aqueous Phase, J. Phys. Chem. B 102 (1998) 7425-7427.

[6] S. Nakata, S. Hiromatsu and H. Kitahata, Multiple Autonomous Motions Synchronized with Complex Formation, J. Phys. Chem. B 107 (2003) 10557-10559.

[7] N. Magome and K. Yoshikawa, Nonlinear Oscillation and Ameba-like Motion in an Oil/Water System, J. Phys. Chem. 100 (1996) 19102-19105.

[8] Y. Sumino, N. Magome, T. Hamada and K. Yoshikawa, Self-Running Droplet: Emergence of Regular Motion from Nonequilibrium Noise, Phys. Rev. Lett. 94 (2005) 068301.

[9] K. Nagai, Y. Sumino, H. Kitahata and K. Yoshikawa, Mode Selection in the Spontaneous Motion of an Alcohol Droplet, Phys. Rev. E 71 (2005) (R)065301.

[10] J. Tersoff, D. E. Jesson and W. X. Tang, Running Droplets of Gallium from Evaporation of Gallium Arsenide, Science 324 (2009) 236-238.

[11] L. Lioubashevski, Y. Hamiel, A. Agnon, Z. Reches and J. Fineberg, Oscillons and Propagating Solitary Waves in a Vertically Vibrated Colloidal Suspension, Phys. Rev. Lett. 83 (1999) 3190-3193.

[12] K. Yoshikawa and N. Magome, Chemomechanical Transduction in an Oil-Water System. Regulation of the Macroscopic Mechanical Motion, Bull. Chem. Soc. Jpn. 66 (1993) 3352 3357.

[13] Y. Hayashima, M. Nagayama and S. Nakata, A Camphor Grain Oscillates while Breaking Symmetry, J. Phys. Chem. B 105 (2001) 5353-5357.

[14] M. Nagayama, S. Nakata, Y. Doi and Y. Hayashima, A Theoretical and Experimental Study on the Unidirectional Motion of a Camphor Disk, Physica D 194 (2004) 151-165. 
[15] Y. Hayashima, M. Nagayama, Y. Doi, S. Nakata, M. Kimura and M. Iida, Self-Motion of a Camphoric Acid Boat Sensitive to the Chemical Environment, Phys. Chem. Chem. Phys. 4 (2002) 1386-1392.

[16] Y. Sumino, H. Kitahata, H. Seto and K. Yoshikawa, Blebbing Dynamics in an Oil-WaterSurfactant System through the Generation and Destruction of a Gel-Like Structure, Phys. Rev. E 76 (2007) (R)055202.

[17] S. Soh, K. J. M. Bishop and B. A. Grzybowski, Dynamic Self-Assembly in Ensembles of Camphor Boats, J. Phys. Chem. B 112 (2008) 10848-10853.

[18] H. Kitahata, N. Yoshinaga, K. H. Nagai and Y. Sumino, Spontaneous Motion of a Droplet Coupled with a Chemical Wave, Phys. Rev. E 84 (2011) (R)015101.

[19] X. Chen, S. Ei and M. Mimura, Self-Motion of Camphor Discs: Model and Analysis, Networks and Heterogeneous Media 4 (2009) 1-18.

[20] M. I. Kohira, Y. Hayashima, M. Nagayama and S. Nakata, Synchronized Self-Motion of Two Camphor Boats, Langmuir 17 (2001) 7124-7129.

[21] Y. Sumino, H. Kitahata, K. Yoshikawa, M. Nagayama, S. M. Nomura, N. Magome and Y. Mori, Chemosensitive Running Droplet, Phys. Rev. E 72 (2005) (R)041603.

[22] N. J. Suematsu, S. Nakata, A. Awazu and H. Nishimori, Celective Behavior of Inanimate Boats, Phys. Rev. E 81 (2010) (R)056210.

[23] E. Heisler, N. J. Suematsu, A. Awazu and H. Nishimori, Collective Motion and Phase Transitions of Symmetric Camphor Boats, J. Phys. Soc. Jpn. 81 (2012) 074605.

[24] E. Heisler, N. J. Suematsu, A. Awazu and H. Nishimori, Swarming of Self-Propelled Camphor Boats, Phys. Rev. E 85 (2012) (R)055201.

[25] H. Kitahata and K. Yoshikawa, Chemo-Mechanical Energy Transduction through Interfacial Instability, Physica D 205 (2005) 283-291.

[26] T. Ohta and T. Ohkuma, Deformable Self-Propelled Particles, Phys. Rev. Lett. 102 (2009) 154101.

[27] T. Ohta, T. Ohkuma and K. Shitara, Deformation of a Self-Propelled Domain in an Excitable Reaction-Diffusion System, Phys. Rev. E 80 (2009) (R)056203.

[28] A. S. Mikhailov and V. Calenbuhr, From Cells to Societies, Springer, Berlin, 2002.

[29] Y. S. Ikura, R. Tenno, H. Kitahata, N. J. Suematsu and S. Nakata, Suppression and Regeneration of Camphor-Driven Marangoni Flow with the Addition of Sodium Dodecyl Sulfate, $J$. Phys. Chem. B 116 (2012) 992-996. 
[30] Suppose that the system has a circular symmetry with respect to the origin. Then, (5) in a steady state becomes Helmholtz equation by change of variables, whose solution along the $x$ axis is expanded by $\mathcal{I}_{n}\left(x / l_{\mathrm{c}}\right)(n=0,1,2,3, \cdots)$ in $\Omega_{\mathrm{c}}$, and $\mathcal{K}_{n}\left(x / l_{\mathrm{c}}\right)(n=0,1,2,3, \cdots)$ in $\Omega \backslash \Omega_{\mathrm{c}}$, where $\mathcal{I}_{n}$ and $\mathcal{K}_{n}$ are the $n$th order modified Bessel functions of the first and second kind, respectively. The characteristic length $l_{\mathrm{c}}$ is called a diffusion length, where $l_{\mathrm{c}}=\sqrt{D / \alpha}$. In (18), the diffusion length is normalized to unity.

[31] H. Kitahata, K. Iida and M. Nagayama, Spontaneous Motion of an Elliptic Camphor Particle, Phys. Rev. E 87 (2013) (R)010901.

[32] Á. Baricz and S. Ponnusamy, On Turán Type Inequalities for Modified Bessel Functions, Proc. Am. Math. Soc. 141 (2013) 523.

[33] G. N. Watson, A Treatise on the Theory of Bessel Functions, Cambridge University Press, 1995. 